\title{
Dark Matter Substructure and Dwarf Galactic Satellites
}

\author{
Andrey Kravtsov \\ Department of Astronomy \& Astrophysics, Kavli Institute for Cosmological Physics, The University of Chicago,
} Chicago, IL 60637, USA

Correspondence should be addressed to Andrey Kravtsov, andrey@oddjob.uchicago.edu

Received 8 June 2009; Accepted 31 August 2009

Academic Editor: Ulrich Hopp

Copyright () 2010 Andrey Kravtsov. This is an open access article distributed under the Creative Commons Attribution License, which permits unrestricted use, distribution, and reproduction in any medium, provided the original work is properly cited.

A decade ago cosmological simulations of increasingly higher resolution were used to demonstrate that virialized regions of Cold Dark Matter (CDM) halos are filled with a multitude of dense, gravitationally bound clumps. These dark matter subhalos are central regions of halos that survived strong gravitational tidal forces and dynamical friction during the hierarchical sequence of merging and accretion via which the CDM halos form. Comparisons with observations revealed that there is a glaring discrepancy between abundance of subhalos and luminous satellites of the Milky Way and Andromeda as a function of their circular velocity or bound mass within a fixed aperture. This large discrepancy, which became known as the "substructure" or the "missing satellites" problem, begs for an explanation. In this paper, the author reviews the progress made during the last several years both in quantifying the problem and in exploring possible scenarios in which it could be accommodated and explained in the context of galaxy formation in the framework of the CDM paradigm of structure formation. In particular, he shows that the observed luminosity function, radial distribution, and the remarkable similarity of the inner density profiles of luminous satellites can be understood within hierarchical CDM framework using a simple model in which efficiency of star formation monotonically decreases with decreasing virial mass satellites had before their accretion without any actual sharp galaxy formation threshold.

\section{Introduction}

In the hierarchical scenario of galaxy formation [1], theoretically rooted in the Cold Dark Matter (CDM) structure formation model [2], galaxies form via cooling and condensation of gas in dark matter halos, which grow via an hierarchical sequence of mergers and accretion. The density perturbations in these models have amplitude that increases with decreasing scale down to $\sim 1$ comoving parsec or below [3], with the smallest fluctuation scale defined by the specific properties of the particles assumed to constitute the majority of the CDM. Smaller perturbations thus collapse first and then grow and merge to form larger and larger objects, with details of the evolution determined by expansion history of the universe (i.e., by parameters describing the background cosmological model) and by the shape of the density fluctuation power spectrum [4].

An example of such evolution in the flat $\Lambda \mathrm{CDM}$ model is illustrated in Figure 1 , which shows collapse of $\mathrm{a} \approx 10^{12} \mathrm{M}_{\odot}$ object. The figure shows that during the early stages of evolution the matter that is incorporated into the final halo collapses into a large number of relatively small clumps with a filamentary, web-like spatial distribution. Further evolution, mediated by the competition between gravity and expansion of space, is a sequence of accretion and mergers that builds objects of progressively larger mass until the single system is formed during the last several billion years of evolution. The figure also shows that cores of some of the small clumps that merge with and are incorporated into larger objects survive until later epochs and are present in the form of halo substructure or subhalos: small dense clumps within virialized regions of larger halos. (Survival of subhalos is not a trivial result and is due to a relatively compact distribution of mass in CDM halos. Ensuring their survival requires a rather large dynamic range in spatial and mass resolution, which had not been achieved until the late 1990s [5-9].)

The CDM model of structure formation is remarkably successful in explaining a wide range of observations from temperature fluctuations of the cosmic microwave background [10] to galaxy clustering and its evolution [11] both qualitatively and, in many cases, quantitatively. Nevertheless, 


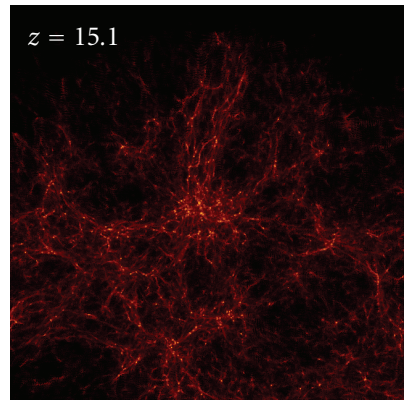

(a)

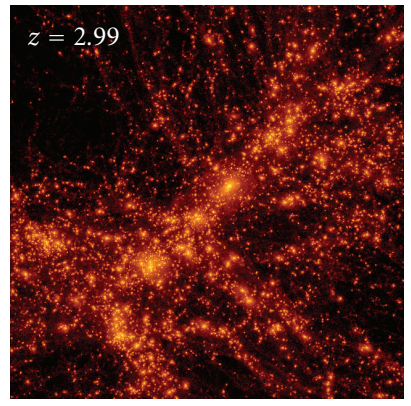

(d)

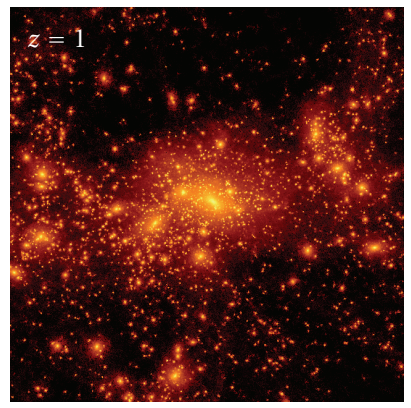

(g)

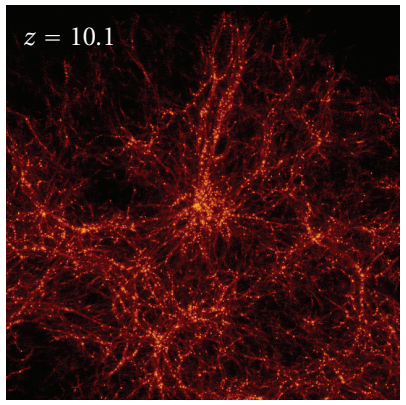

(b)

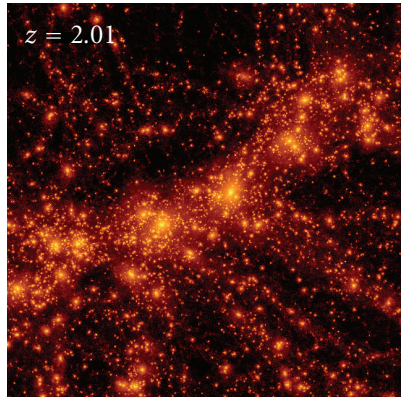

(e)

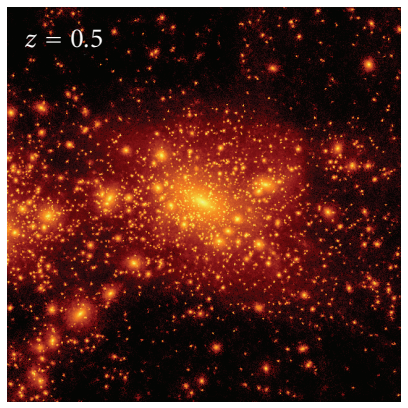

(h)

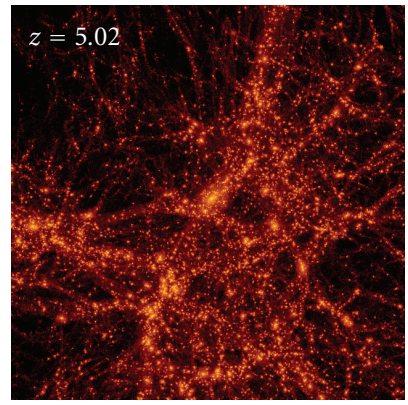

(c)

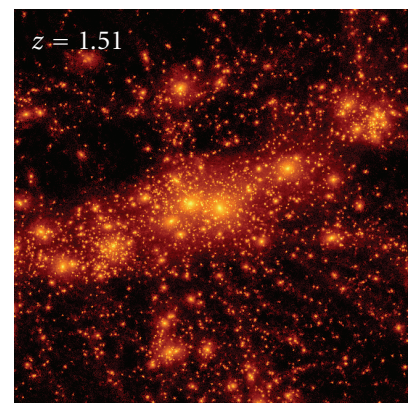

(f)

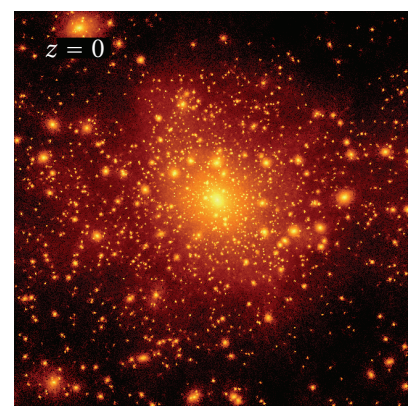

(i)

FIGURE 1: Formation of a Milky Way-sized dark matter halo in a cosmological simulation of flat $\Lambda$ CDM $\operatorname{cosmology}\left(\Omega_{\mathrm{m}}=1-\Omega_{\Lambda}=0.3\right.$, $h=0.7, \sigma_{8}=0.9$ ). The panels show an evolutionary sequence at nine redshifts (from left to right and from top to bottom) focusing on the most massive progenitor of the main halo at each epoch (redshift of each epoch is shown in the left upper corner). The rendering shows the dark matter particles with intensity indicating the local matter density on a logarithmic stretch. The build-up of the halo proceeds through a series of spectacular mergers, particularly frequent in the early stages of evolution. Many of the merging clumps survive until the present epoch $(z=0)$ in the form of "substructure." The size of the region shown is about 3 comoving Mpc at $z=15$, monotonically zooming in to a scale of $\approx 1$ comoving Mpc across at $z=0$.

many key details of the model are still being developed $[12,13]$ and its testing is by no means complete.

One area of active investigation is testing predictions of the CDM models at scales from a few kpc to tens of pc (i.e., the smallest scales probed by observations of galaxies). In particular, there is still tension between predictions of the central mass distribution in galaxies $[14,15]$ and sizes and angular momenta of galactic disks and observational results $[16,17]$. Notably, this tension has not gone away during the past 10-15 years, even though both theoretical models and observations have improved dramatically.

Another example of tension between CDM predictions and observations that has been actively explored during the last decade is the fact that satellite systems around galaxies of different luminosity are qualitatively different, even though their dark matter halos are expected to be approximately scaled down versions of each other [18], with their total mass as the scaling parameter. Faint dwarf galaxies usually have no luminous satellites at all, Milky Way and Andromeda have a few dozen, but clusters of galaxies often have thousands of satellites around the brightest cluster galaxy.

The number of gravitationally bound satellite subhalos at a fixed mass relative to the mass of their host CDM halo, on the other hand, is expected to be approximately the same $[8,19,20]$. This is illustrated in Figure 2, which shows distribution of dark matter out to approximately two virial radii around the centers of two CDM halos of masses different by two orders of magnitude. It is clear that it is 


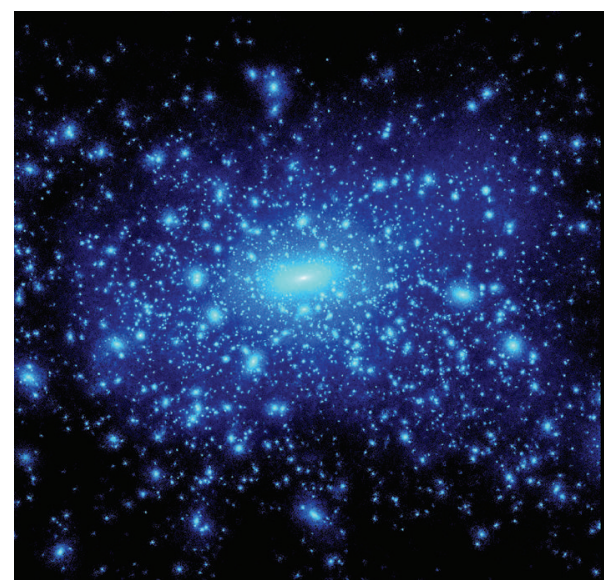

(a)

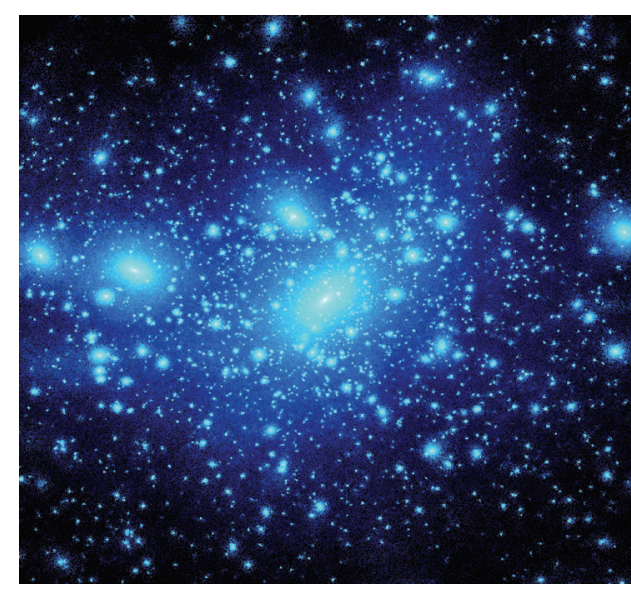

(b)

Figure 2: Comparison of two $z=0$ halos of masses $3 \times 10^{14} M_{\odot}$ and $3 \times 10^{12} M_{\odot}$ formed in flat $\Lambda$ CDM cosmology $\left(\Omega_{\mathrm{m}}=1-\Omega_{\Lambda}=0.3\right.$, $\left.h=0.7, \sigma_{8}=0.9\right)$. In each case the mass distribution around the center of the halo is shown to approximately two virial radii from the center of each halo. Both objects were resolved with similar number of particles and similar spatial resolution relative to the virial radius of the halo in their respective simulations. I leave it as an exercise to the reader to guess the mass of the halo shown in each panel.

not easy to tell the mass of the halo by simply examining the overall mass distribution or by counting the number of subhalos. This is a visual manifestation of approximate (but not exact, see, e.g., [21, 22]) self-similarity of CDM halos of different mass. If we would compare similar images of distribution of luminous matter around galaxies and clusters, the difference would be striking.

The manifestly different observed satellite populations around galaxies of different luminosities and expected approximately self-similar populations of satellite subhalos around halos of different mass is known as the substructure problem $[8,23,24]$. In the case of the best studied satellite systems of the Milky Way and Andromeda galaxies, the discrepancy between the predicted abundance of small-mass dark matter clumps and the number of observed luminous satellites as a function of circular velocity (see Section 2) has been also referred to as the "missing satellites problem." (The name derived from the title "Where are the missing galactic satellites?" of one of the papers originally pointing out the discrepancy [24].) The main goal of this paper is to review theoretical and observational progress in quantifying and understanding the problem over the last decade.

\section{Quantifying the Substructure and Luminous Satellite Populations}

In order to connect theoretical predictions and observations on a quantitative level, we need descriptive statistics to characterize population of theoretical dark matter subhalos and observed luminous satellites. Ideally, one would like theoretical models to be able to predict properties of stellar populations hosted by dark matter halos and subhalos and make comparisons using statistics involving directly observable quantities, such as galaxy luminosities. In practice, however, this is difficult as such predictions require modeling of still rather uncertain processes shaping properties of galaxies during their formation. In addition, the simulations can reach the highest resolution in the regime when complicated and computationally costly galaxy formation processes are not included and all of the matter in the universe is modeled as a uniform collisionless and dissipationless component (i.e., the component that cannot dissipate the kinetic energy it acquires during gravitational collapse and accompanying gravitational interaction and relaxation processes). Such simulations thus give the most accurate knowledge of the dark matter subhalo populations, but can only predict dynamical subhalo properties such as the depth of their potential well or the total mass of gravitationally bound material. Therefore, in comparisons between theoretical predictions and observations so far, the most common strategy was to find a compromise quantity that can be estimated both in dissipationless simulations and in observations.

2.1. Quantifying the Subhalo Populations. Starting with the first studies that made such comparisons using results of numerical simulations $[8,24]$ the quantity of choice was the maximum circular velocity, defined as

$$
V_{\max }=\max \left(\frac{G m(<r)}{r}\right)^{1 / 2},
$$

where $m(<r)=4 \pi \int \rho(r) r^{2} d r$ is the spherically averaged total mass profile about the center of the object. $V_{\max }$ is a measure of the depth of the potential (the potential energy of a self-gravitating system is $W \propto V_{\max }^{2}$ ) and can be fairly easily computed in a cosmological simulation once the center of a subhalo is determined. (The detailed description of the procedure of identifying the centers of subhalos is beyond the scope of this paper, but is nevertheless pertinent. While many different algorithms are used in the literature [6, 9, 25-29], all algorithms boil down to the automated search for density peaks (most often in configuration space, but sometimes 
in the phase space) field smoothed at a scale comparable to or smaller than the size of the smallest subhalos in the simulations. Once the peaks are identified, the gravitationally bound material around them is usually found by iteratively removing the unbound particles.) The attractive feature of $V_{\text {max }}$ is that it is well defined and does not require estimate of a physical boundary of subhalos, which is often hard to determine. The price is that resolution required to get the $V_{\max }$ correctly for a subhalo is higher, compared, for example, to the total bound mass of subhalo, because $V_{\max }$ is probing the mass distribution in the inner regions of halos.

The total gravitationally bound mass of a subhalo, $m_{\text {sub }}$, is less sensitive to the resolution, but requires careful separation between real subhalo particles and unbound particles from the diffuse halo of the host dark matter halo. This can be quite difficult in the inner regions of the host system where density of the background diffuse halo is comparable to the internal density of subhalo or when two subhalos overlap substantially.

An alternative option is to define mass of a subhalo within a fixed physical radius. For suitably chosen radius value, the mass can be measured unambiguously both in simulations and in observations. We will discuss the measurement of the enclosed mass and comparisons between simulations and observations below in Sections 3 and 4.3 (see Figures 8 and 12).

Figure 3 shows the cumulative circular velocity and mass functions (CVF and CMF) of subhalos within the virial radius (defined as $R_{\Delta}=\left(3 M_{\Delta} / 4 \pi \Delta \bar{\rho}\right)^{1 / 3}$, where $\bar{\rho}$ is the mean matter density in the universe and $\Delta=337$, corresponding to the $z=0$ virial overdensity suggested by the spherical collapse model in the $\Lambda$ CDM cosmology [30]) of a simulated Milky Way sized halo, formation of which was illustrated in Figure 1. Both cumulative functions can be approximated by power laws over the ranges of circular velocity and in units of $V_{\max }$ and virial mass of the host: $v \equiv V_{\max } / V_{\max }^{\text {host }} \lesssim 0.1$ and $\mu \equiv m_{\text {sub }} / M_{\text {vir }}^{\text {host }} \lesssim 0.001$ with the slopes of $-2.7 \div-3$ and $\approx-0.8 \div-0.9$, respectively. At large circular velocities deviations from the power law can be significant due to small numbers of subhalos.

The highest-resolution simulations (to date) of the individual MW-sized DM halos formed in the concordance $\Lambda \mathrm{CDM}$ cosmology [31-33] show that the power laws with the slopes in the range indicated above describe the CVF and CMF down to $\mu \approx 10^{-7}$ and $\nu \approx 10^{-2}$. Note, however, that over a wider range of subhalo masses the power law can be expected to change slowly reflecting the changing slope of the rms fluctuations as a function of scale, which controls the abundance of halos as a function of mass [34, 35].

The amplitude of the mass and velocity functions is sensitive to the normalization of the power spectrum on small scales $[31,36,37]$ and is thus sensitive to the cosmological parameters that control the normalization (such as tilt and normalization $\sigma_{8}$ ).

For a given cosmology, the normalization of the CVF and CMF scales approximately linearly with the host halo mass [19]: $N\left(m_{\text {sub }} \mid M_{\mathrm{h}}\right) \propto M_{\mathrm{h}}$. The halo-to-halo scatter in the normalization of CVF and MCF for a fixed host halo virial mass is described by the Poisson distribution [19]: $\sigma_{N(>\mu)}=$

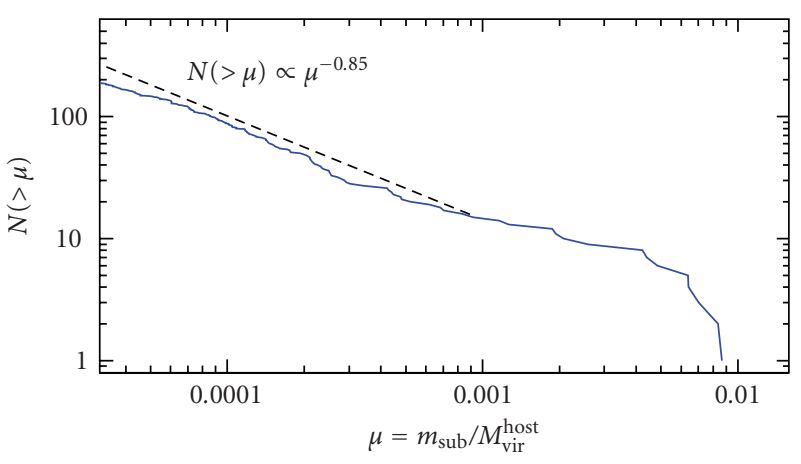

(a)

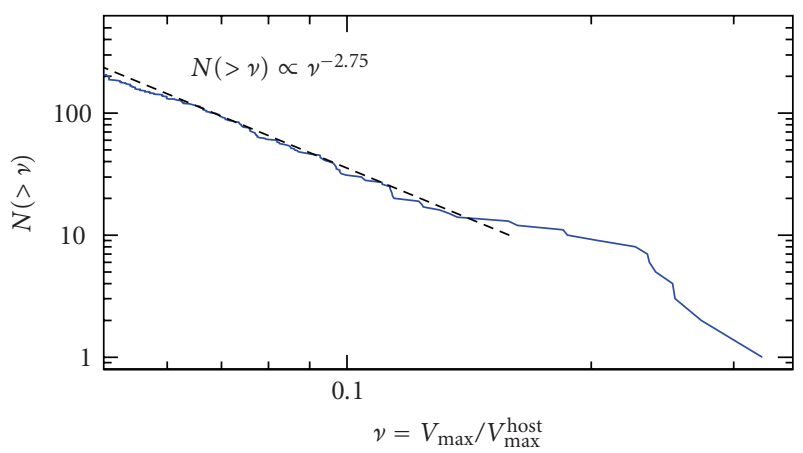

(b)

FIGURE 3: Cumulative circular velocity and mass function of subhalos within the virial radius $R_{337}=328 \mathrm{kpc}$ of a halo of virial mass $M_{337}=2 \times 10^{12} M_{\odot}$ at $z=0$. The dashed lines show power laws with the slopes indicated in the legend of each panel.

$\sqrt{N(>\mu)}$. The fractional scatter is, therefore, quite small for small $\mu$ and $\nu$ (large $N)$.

The mass and circular velocity functions within a given radius describe the overall abundance of subhalos of different mass, but not their radial distribution. The latter depends rather sensitively on how the subhalo samples are selected [38]. This is because subhalos at different distances from their host halo center on average experience different tidal mass loss, which affects different subhalo properties by different amount. Subhalo mass is the most affected quantity as large fraction of halo mass when it accretes is relatively loosely bound and is usually lost quickly. Although circular velocity is determined by the inner mass distribution in the inner mass of subhalos, it is still affected by tidal stripping (albeit to a less degree and slower than the total mass [39]).

The average mass loss experienced by subhalos increases with decreasing distance to the center of the host halo [38]. Therefore, selecting subhalos based on their current bound mass or circular velocity biases the sample against subhalos at smaller radii and results in the radial distribution much less concentrated than the overall mass distribution of the host halo $[6,26,38-42]$. Conversely, one can expect that if the selection of subhalos is made using a quantity not affected by stripping, the bias should be smaller or even disappear altogether. 
Figure 4(a) shows the radial distribution of subhalos (the same population as in Figure 3) selected using their current bound mass or circular velocity and density profile of dark matter within an MW-sized host halo. The figure shows that the subhalo distribution is less radially concentrated compared to the overall density profile because selection using current subhalo properties affected by tidal evolution biases the sample against the inner regions. Figure 4(b) shows the radial distribution of subhalos in the same host halo but now selected using circular velocity and mass the subhalos had before accretion (which are of course not affected by the tides). In this case, the radial profile is very close to that of the dark matter distribution. This dependence of the radial profile on the property used for subhalo selection should be kept in mind when the observed and predicted radial distributions are compared. The latter are selected based on their luminosity (i.e., the stellar mass), which may be affected by tides much less than either the total bound mass or circular velocity [38, 43].

Finally, the spatial distribution of satellites is not completely spherically symmetric, but is triaxial, which reflects their accretion along filaments and subsequent evolution in the triaxial potential of their host halos [44-46].

2.2. Quantifying Populations of Luminous Galactic Satellites. Although we currently know only a few dozens of nearby satellite galaxies around the Milky Way and Andromeda, these galaxies span a tremendous range of the stellar densities and luminosities. The two brightest satellites of the Milky Way, the Large and Small Magellanic Clouds (LMC and SMC), are easily visible by the naked eye in the southern hemisphere and have, therefore, been known for many hundreds of years, while the faintest satellites have been discovered only very recently using sophisticated search algorithms and the vast data sets of stellar photometry in the Sloan Digital Sky Survey and contain only a few hundred stars [47, 48]. Up until the late 1990s, only a dozen dwarf galaxies were known to exist within $300 \mathrm{kpc}$ of the Milky Way, with a similar number around the Andromeda [49]. These galaxies have luminosities $L \gtrsim 10^{5} L_{\odot}$ and morphologies of the three types: (1) dwarf irregular galaxies (dIrrs, e.g., LMC and SMC) - low surface brightness galaxies of irregular appearance which have substantial amount of gas and thus exhibit continuing star formation, (2) dwarf spheroidal galaxies (dSphs, e.g., Draco or Fornax)—low surface brightness galaxies with spheroidal distribution of stars and no (or very little) ongoing star formation, and (3) dwarf elliptical galaxies (dEs, e.g., M32)—high-surface brightness, low-luminosity ellipticals with no gas and no current star formation. $\mathrm{dSph}$ and $\mathrm{dE}$ galaxies tend to be located within $200 \mathrm{kpc}$ of their host galaxies, while dIrr galaxies are distributed more uniformly. This tendency is called the "morphological segregation" [49] and appears to exist in other nearby groups of galaxies [50]. The properties of these "classical" dwarf galaxies are reviewed extensively by [49] (see also recent study of scaling relations of dwarf galaxies by [51]).

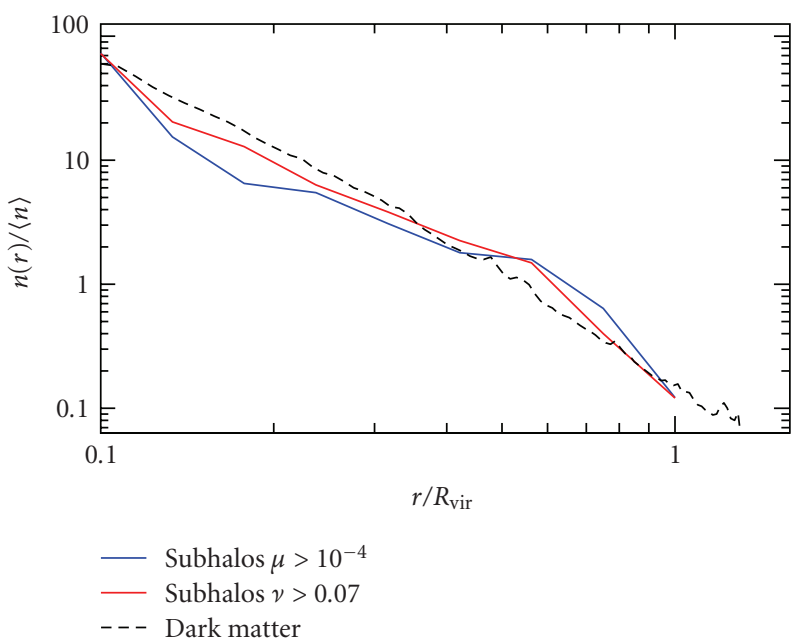

(a)

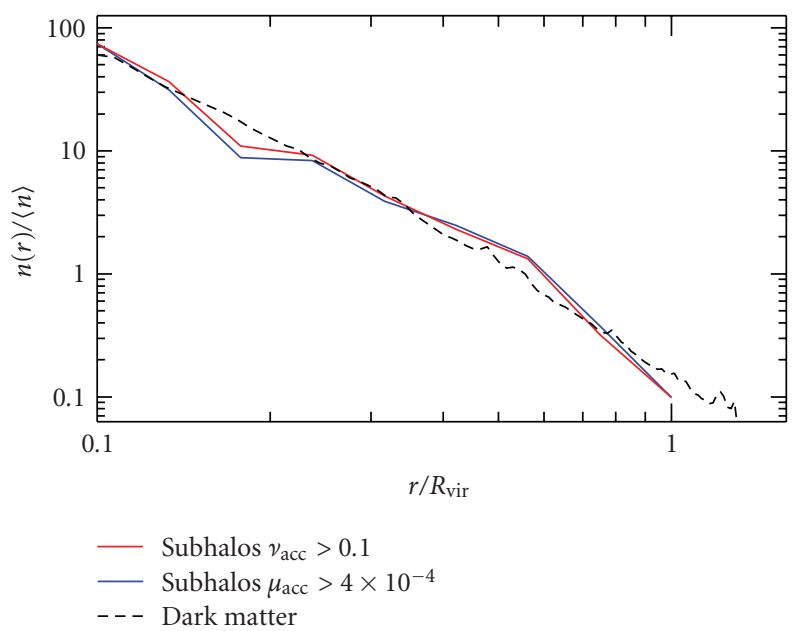

(b)

FIGURE 4: Radial distribution of subhalos (solid lines) selected using their different properties ( $V_{\max }$ and total mass - solid red and blue lines, resp.) compared to the matter density profile in their host halo (dashed lines). (a) shows the profiles for subhalos selected using circular velocity and bound mass a the current epoch, while the lower shows radial distribution of subhalos selected using the corresponding quantities before subhalo was accreted unaffected by the subsequent tidal mass loss. Note that minimum threshold values for subhalo selection, $\mu_{\min }$ and $\nu_{\min }$, are different in (b) because for a typical mass loss many subhalos with smaller circular velocities and masses at the time of accretion fall below the completeness limit of the simulation by $z=0$.

Despite the wide range of observed properties, all of the nearby dwarfs share some common features in their star formation histories (SFHs). The SFHs of all classical dwarfs are characterized by a rather chaotically varying star formation rates. Most bright dwarfs form stars throughout their evolution, although the majority of stars may be formed in several main episodes spread over ten or more billion years [52-55], and have at least some fraction of stars that formed in the first two billion years of the evolution of the universe. In terms of their SFHs, the main difference between the dwarf 


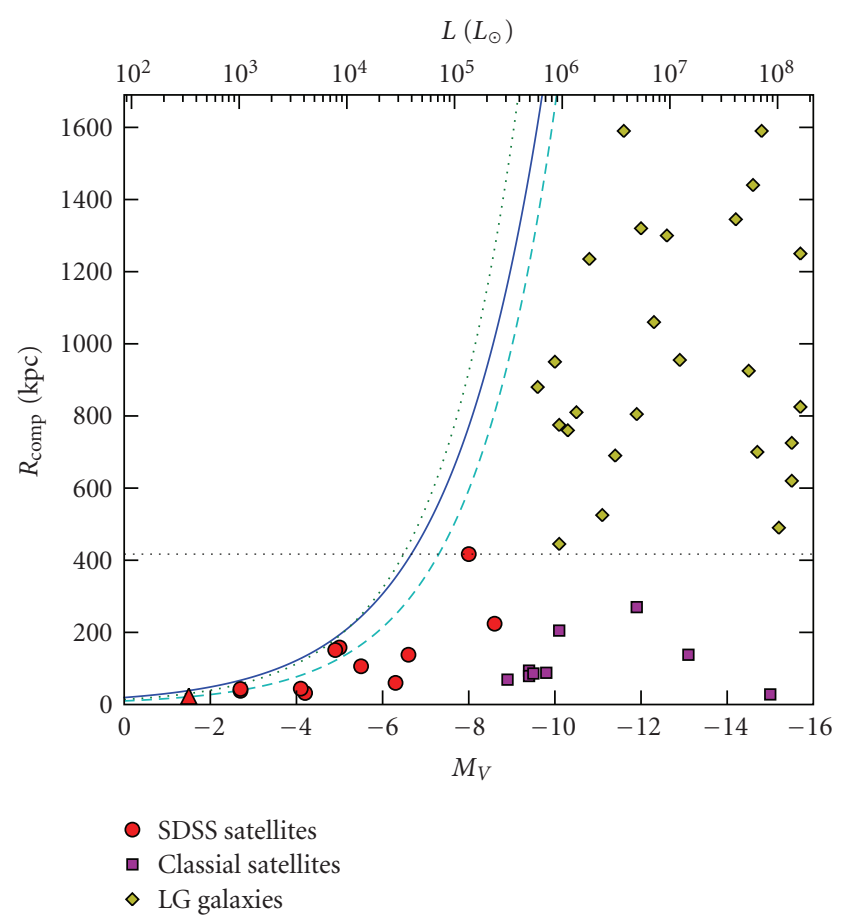

Figure 5: The distance to which the current samples of dwarf satellite galaxies around the Milky Way are complete (dotted, solid and dashed lines correspond to somewhat different assumptions about detection limits of dwarf galaxies, see [56] for details) as a function of galaxy luminosity (absolute V-band magnitude on the bottom scale and physical luminosity in units of solar luminosity at the top scale). The points show different types of observed satellites around the Milky Way and in the Local Group. The horizonthal dotted line indicates the outer radius for satellites counts shown in the Figure 6. Note that the completeness distance of the faintest recently discovered satellites is $\lesssim 50 \mathrm{kpc}$. Adopted from [56].

irregular and dwarf spheroidal galaxies is in the presence or absence of star formation in the last two billion years [54].

The radial distribution of the classical satellites around the Milky Way is rather compact. For the dwarf galaxies within $250 \mathrm{kpc}$ of the Milky Way, the median distance to the center of the Galaxy is $\approx 70 \mathrm{kpc}[39,57]$, while the predicted median distance for subhalos is $\approx 120-140 \mathrm{kpc}$ [39] (see Figure 13). The distribution of the satellites around the Andromeda galaxies is consistent with that of the Milky Way satellites, but is less accurately determined due to larger errors in distances. The spatial distribution of satellites about the Milky Way and Andromeda is also manifestly nonisotropic with the majority of the satellites found in a flattened structure nearly perpendicular to the disk [58-62].

In 1994, a new faint galaxy was discovered in the direction toward the center of the Galaxy (in the Sagittarius constellation [63]). The galaxy is similar to other nearby dwarf spheroidal galaxies in its properties but is remarkably close to the solar system (the distance of only $\approx 28 \mathrm{kpc}$ ) and is in the process of being torn apart by the tidal interactions with the Milky Way. This interaction has produced a spectacular tidal tail, which has wrapped several times along the orbit of the Sagittarius dwarf [64].

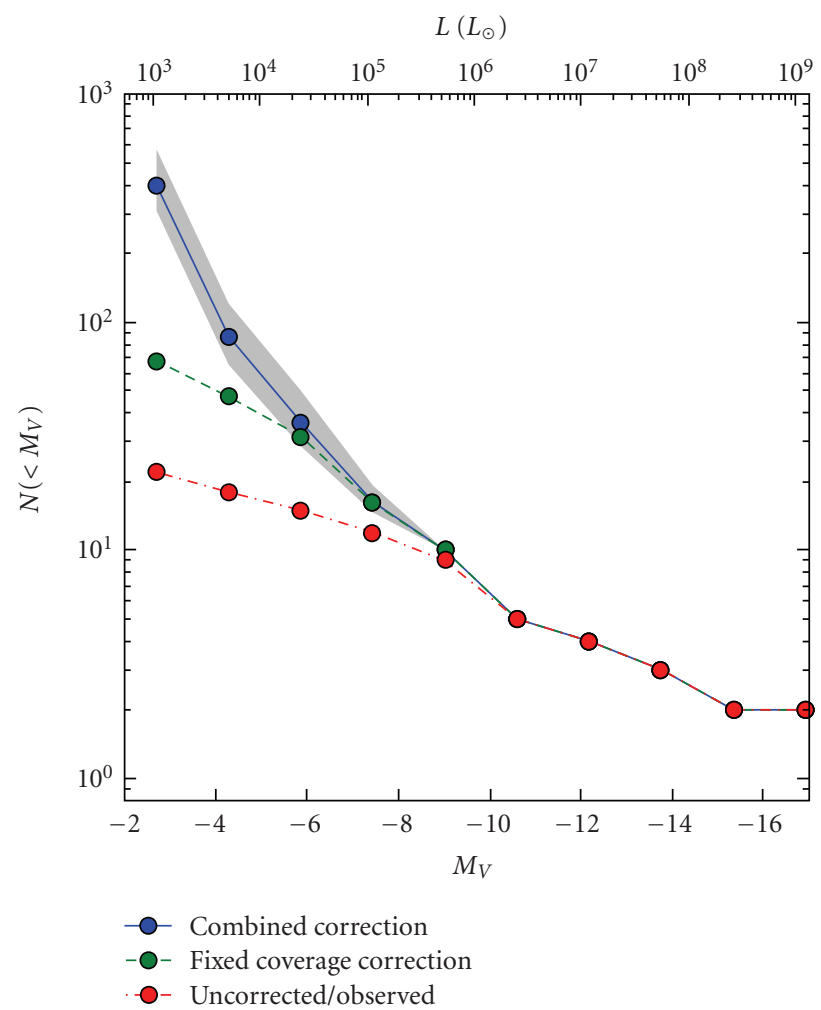

FIGURE 6: The luminosity function of dwarf galaxies around the Milky Way. The function includes all observed galaxies within $417 \mathrm{kpc}$ of the Milky Way. The red circles connected by the dotted line show the luminosity function as observed, without any corrections for incompleteness. The green circles connected by the dashed line show the observed luminosity function corrected for limited area coverage of surveys on the sky. The blue circles connected by the solid line show luminosity function corrected for the radial bias using radial distribution of subhalos from the Via Lactea I simulation. Adopted from [56].

The discovery of this new satellite has alerted researchers in the field to the possibility that other satellites may be lurking undiscovered in our cosmic neighborhood. The advent of wide field photometric surveys, such as the Sloan Digital Sky Survey and the targeted surveys of regions around the Andromeda galaxy, and new search techniques has resulted in dozens of new satellite galaxies discovered during the last decade $[47,48,65-71]$ with many more discoveries expected in the near future [56, 72]. The majority of the newly discovered galaxies are fainter than the "classical dwarfs" known prior to 1998. Due to their extremely low luminosities (as low as $\sim 1000 L_{\odot}$ in the case of the Segue 1 [48]), they have collectively been referred to as the "ultrafaint" dwarfs. Such low luminosities (and implied stellar masses) indicate an extreme mode of galaxy formation, in which the total population of stars produced during galaxy evolution is smaller than a star cluster formed in a single star formation event in more luminous galaxies. (Alternatively, extremely low luminosities of the ultra-faint dwarfs can also be explained as a highly stripped remnants of more luminous dwarfs [43]. While this possibility is not excluded, it is 
disfavored by the fact that ultra-faint dwarfs appear to lie on the continuation of the luminosity-metallicity relation of more luminous dwarf galaxies [73].)

More practically, the extreme faintness of the majority of dwarf satellites implies that we have a more or less complete census of them only within the volume of $\sim 30-50 \mathrm{kpc}$ of the Milky Way $[56,74]$. Figure 5 shows the distance to which the dwarfs of a given luminosity are complete in the SDSS survey, in which the faintest new dwarfs have been discovered. The figure shows that we have a good census of the volume of the Local Group only for the relatively bright luminosities of the "classical" satellites. At the fainter luminosities of the ultra-faint dwarfs, on the other hand, we can expect to find many more systems at larger radii in the future deep wide area surveys. The exact number we can expect to be discovered depends on their uncertain radial distribution, but given the numbers of already discovered dwarfs and our current knowledge of the radial distribution of brighter satellites (and expected radial distribution of subhalos), we can reasonably expect that at least a hundred faint satellites exist within $400 \mathrm{kpc}$ of the Milky Way. This is illustrated in Figure 6, which shows the luminosity function of the Milky Way satellites corrected for the volume not yet surveyed under different assumptions about radial distribution of the satellites [56].

The basis for considering these extremely faint stellar systems as bona fide galaxies is the fact that unlike star clusters, they are dark matter dominated: that is, the total mass within their stellar extent is much larger than the stellar mass expected for old stellar populations [48]. The total dynamical masses of these galaxies are derived using kinematics of stars. (These faint dwarf spheroidal galaxies do not have cold gas and therefore their mass profiles cannot be measured using the gas rotation curve, as is commonly done for more massive dIrr galaxies.) Highresolution spectroscopy of the red giant stars in the vicinity of each galaxy provides the radial velocities of these stars. The radial velocities can then be modeled using using the Jeans equilibrium equations to derive the total mass profile [75-80]. This modeling requires certain assumptions about the unknown shape of the stellar distribution and velocity distribution of stars, as well as assumptions about the shape and radial profile of the dark matter distribution. The resulting mass profile, therefore, has some uncertainty associated with these assumptions $[75,78,80]$.

Additionally, the ultra-faint dwarfs follow scaling relations of the brighter classical satellites such as the luminositymetallicity relation [73] and, therefore, seem to be the low luminosity brethren within the family of dSph galaxies.

\section{Defining the Substructure Problem}

As I noted above, comparison of theory and observations in terms of the directly observable quantities such as luminosities is possible only using a galaxy formation model. These models, although actively explored [39, 81-87] (see also Section 4.3) are considerably more uncertain than the predictions of dissipationless simulations on the properties

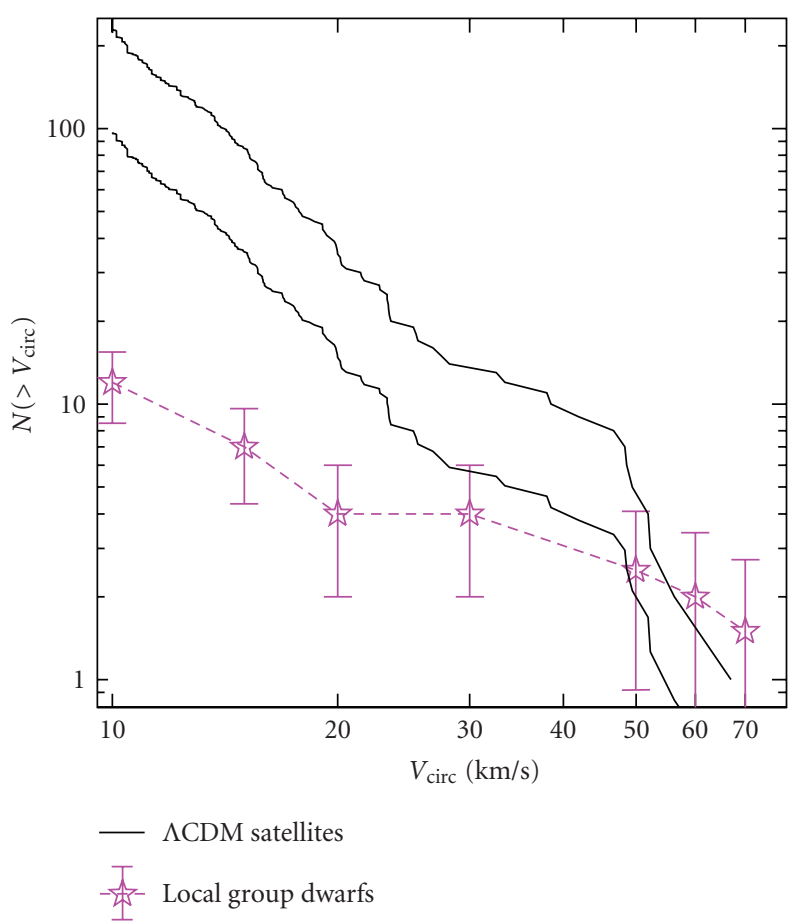

FIGURE 7: Comparison of the cumulative circular velocity functions, $N\left(>V_{\max }\right)$, of subhalos and dwarf satellites of the Milky Way within the radius of $286 \mathrm{kpc}$ (this radius is chosen to match the maximum distance to observed satellites in the sample and is smaller than the virial radius of the simulated halo, $R_{337}=326 \mathrm{kpc}$ ). The subhalo VFs are plotted for the host halos with maximum circular velocities of $160 \mathrm{~km} / \mathrm{s}$ and $208 \mathrm{~km} / \mathrm{s}$ that should bracket the $V_{\max }$ of the actual Milky Way halo. The VF for the observed satellites was constructed using circular velocities estimated from the line-of-sight velocity dispersions as $V_{\max }=\sqrt{3} \sigma_{r}$ (see the discussion in the text for the uncertainties of this conversion).

of dark matter subhalos. Given that observed dwarf satellites are very dark matter dominated, the dissipative processes leading to formation of their stellar component are expected to have a limited effect on the distribution of the dynamically dominant dark matter. Fruitful comparison between simulation predictions and observations is, therefore, possible if a quantity related to the total mass profile can be measured in the latter.

The first attempts at such comparisons $[8,9]$ assumed isotropy of the stellar orbits and converted the line-ofsight velocity dispersion of stars in $\mathrm{dSph}$ satellites, $\sigma_{r}$, to estimate their maximum circular velocities as $V_{\max }=$ $\sqrt{3} \sigma_{r}$. The admittedly oversimplistic conversion was adopted simply due to a lack of well-measured velocity profiles and corresponding constraints on the mass distribution at the time. Figure 7 shows such a comparison for the classical satellites of the Milky Way and subhalo populations in Milky Way-sized halos formed in the concordance $\Lambda$ CDM cosmology.(I did not include the new ultra-faint satellites in the comparison both because their $V_{\max }$ values are much more uncertain and because their total number within the virial radius requires uncertain corrections from the 
currently observed number that probes only the nearest few dozen kpc. The velocity dispersions of the ultra-faint dwarfs are very similar to each other $(\sim 5 \mathrm{~km} / \mathrm{s})$ and they, therefore, formally have similar $V_{\max }$ values according to this simple conversion method (hence, they would all be "bunched up" at about the same $V_{\max } \sim 9 \mathrm{~km} / \mathrm{s}$ value). The maximum circular velocity of the halos of these galaxies is expected to be reached at radii well beyond the stellar extent and its estimate from the observed velocity dispersions requires substantial extrapolation and assumptions about the density profile outside the radii probed by stars. The errors of the derived values of $V_{\max }$ can, therefore, be quite substantial $[75,88]$. I will compare the predicted luminosity function of the luminous satellites using a simple galaxy formation model in Section 4.3 (see Figure 11).)

The observed velocity function is compared to the predicted VF of dark matter subhalos within a $286 \mathrm{kpc}$ radius of Milky Way-sized host halos. In literature, the term "Milky Way-sized" is often used to imply a total virial mass of $M_{\mathrm{vir}} \approx 10^{12} M_{\odot}$ and maximum circular velocity of $V_{\max } \approx 200 \mathrm{~km} / \mathrm{s}$. However, there is some uncertainty in these numbers. Therefore, the figure shows the VFs for the host halos with $V_{\max }=208 \mathrm{~km} / \mathrm{s}$ and $160 \mathrm{~km} / \mathrm{s}$. The former is measured directly in a simulation of the halo of that circular velocity, while the latter VF was rescaled as $N\left(>V_{\max }\right) \propto M_{\mathrm{vir}, 1} / M_{\mathrm{vir}, 2}=\left(V_{\max , 1} / V_{\max , 2}\right)^{3.3}$, using scaling measured statistically in the simulations [19].

The simple conversion of $\sigma_{r}$ to $V_{\max }$ has justly been criticized as too simplistic [89]. Indeed, the conversion factor $\eta \equiv V_{\max } / \sigma_{r}$ requires a good knowledge of mass profile from small radii to the radius $r_{\max }$. The mass profile derived from the Jeans equation has errors associated with uncertainties in the anisotropy of stellar orbits, as well as with uncertainties of spatial distribution of stellar system and/or its dynamical state [76, 90]. Most importantly, the mass profile is only directly constrained within the radius where stellar velocities are measured, $r_{*}$. If this radius is smaller than $r_{\max }$, conversion factor $\eta$ depends on the form of the density profile assumed for extrapolation. The uncertainties of the derived mass profile within the stellar extent will of course also be magnified increasingly with increasing $r_{\max } / r_{*}$ ratio [90].

Thus, for example, Stoehr et al. [89] have argued that the conversion factor can be quite large $(\eta \gtrsim 2-4)$ if the density profile is shallow in the inner regions probed by the stars. Such large conversion factor would shift the low $V_{\max }$ points in Figure 7 to the right closer to the subhalo VF $[78,89,91]$ and would imply that there is a sharp drop in the luminosity function of satellites below a certain threshold circular velocity $\left(V_{\max } \approx 30 \mathrm{~km} / \mathrm{s}\right.$ ). High-resolution simulations of individual satellites, on the other hand, have demonstrated that the CDM satellites retain their cuspy inner density profiles, even as they undergo significant tidal stripping [92]. (The profiles can be even steeper than predicted by dissipationless simulations due to the effects of adiabatic contraction during gas condensation towards the center of the dwarf progenitors [93]. Although the effect of baryon condensation in dwarfs are uncertain at present, one can argue that they can reasonably be expected to be modest, given large mass-tolight ratios of faint dwarfs [48].) This implies that $\eta$ is likely not as large as advocated by Stoehr et al. The main uncertainty in its actual value for a specific satellite is then due to the uncertainty in the density profile and ratio $r_{\max } / r_{*}$.

Recently, Peñarrubia et al. [78] combined the measurements of stellar surface density and $\sigma_{r}(R)$ profiles to estimate $V_{\max }$ values for individual observed MW satellites under the assumption that their stellar systems are embedded into NFW dark matter potentials. Such procedure by itself does not produce a reliable estimate of $V_{\max }$, because NFW potentials with a wide range of $V_{\max }$ can fit the observed stellar profiles. To break the degeneracy, Peñarrubia et al. have used the relation between $V_{\max }$ and $r_{\max }$ expected in the concordance $\Lambda \mathrm{CDM}$ cosmology. They showed that this results in estimates of $V_{\max }$ which imply $\eta \approx 2$ 3 [43, Figure 4]. This estimate does not take into account effects of tidal stripping on the evolution of the $r_{\max }-V_{\max }$ relation. Typically, subhalos located in the inner regions of the halo are expected to have lost $\sim 60 \%-90 \%$ of their initial mass by $z=0$ due to tidal stripping [22, 38, 39]. For such mass loss $V_{\max }$ changes only by $\approx 20 \%-30 \%[39,78]$ but $r_{\max }$ should change by a factor of $\approx 2-3[43,92]$.

In a subsequent study, Peñarrubia et al. [43] used controlled simulations of subhalo evolution to argue that tidal stripping does not significantly affect their inferred conversion factor $\eta$ [43, Figure 9]. This conclusion, however, was drawn based on the systems in which both stellar system and DM halo were significantly stripped. In such system, $r_{\max }$ is close to the stellar radius and $\sigma_{0}$ and $V_{\max }$ evolve in sync. For systems with more realistic mass loss and with stars deeply embedded within $r_{\max }$, however, stellar system (and $\left.\sigma_{0}\right)$ may not be affected, while $V_{\max }$ can evolve significantly. For such systems, the method of [78] will lead to a significant overestimate of $\eta$. Indeed, the systems in [43, Figure 9] for which the method overestimates $\eta$ (by a factor of $\approx 1.4$ ) the most are the systems with moderate total mass loss and least affected stellar systems. Note that even these systems have likely experienced more tidal loss than most of the real dSph satellites.

Another factor in estimates of $V_{\max }$ is anisotropy of stellar velocities in dSph (e.g., [92]). For example, recent analysis of observed velocity dispersion profiles of "classical" dSph by [79], in which the anisotropy of stellar orbits was treated as free parameters, results in estimates of $V_{\max }$ of their host subhalos in the range $\sim 10-25 \mathrm{~km} / \mathrm{s}$, smaller than would be suggested if correction factor was $\eta \approx 2-3$.

Regardless of the actual conversion factor value, however, it is clear that it cannot change the main difference between the observed and predicted VFs - the large difference in their slope-unless $\eta$ strongly depends on $V_{\max }$ (there is no observational evidence for this so far).

Another promising approach is to abandon attempts to derive $V_{\max }$ altogether and to measure instead the observed mass within the radius where the uncertainty of the measured mass profile is minimal. Such radius is close to the stellar extent of observed galaxies [78, 80, 88, 90]. Figure 8, adopted from [90], shows comparison of the mass functions of subhalos in the Via Lactea I simulation [94] 


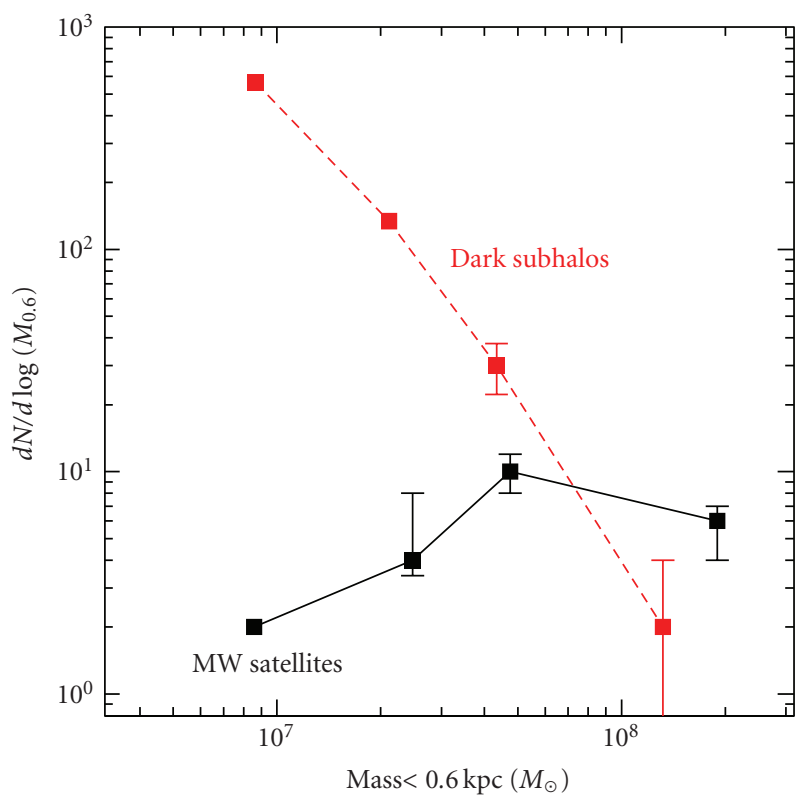

FIGURE 8: The mass function of dwarf satellites of the Milky Way, where masses of subhalos and observed satellites are measured within a fixed physical radius of $0.6 \mathrm{kpc}$. Adopted from [90].

and observed satellites of the Milky Way, where masses are measured within a fixed physical radius of $600 \mathrm{pc}$ (see also the discussion in Section 4.3 and Figure 12). The figure shows that the simulated and observed mass functions are different, the conclusion similar to that derived from the comparison of the circular velocity functions.

Thus, the discrepancy that is clearly seen in the comparison of circular velocity functions, measured with more uncertainty in observations, persists if the comparison is done using a much better measured quantity. Unfortunately, the stellar distribution in most of the newly discovered ultrafaint dwarf galaxies does not extend out to $600 \mathrm{pc}$ radius and $m(r<0.6 \mathrm{kpc}) \equiv m_{0.6}$, therefore, cannot be measured as reliably for these faint systems as for the classical dwarfs. Similar comparisons have to be carried out using masses within smaller radii. This puts stringent requirements on the resolution of the simulations, as they need to reliably predict mass distribution of subhalos within a few hundred parsec radius. Such high-resolution simulations are now available [31-33].

We can draw two main conclusions from the comparisons of the circular velocity functions and the more reliable $m_{0.6}$ mass functions of subhalos and observed satellites presented in the previous sections, even taking into account existing uncertainties in deriving circular velocities and the total dynamical masses for the observed satellites. First, the predicted abundance of the most luminous satellites is in reasonable agreement with the data, even though the statistics are small. Most MW-sized halos simulated in the concordance $\Lambda \mathrm{CDM}$ cosmology have $\sim 1$ SMC/LMC sized $\left(V_{\max } \approx 50-70 \mathrm{~km} / \mathrm{s}\right.$ ) subhalos within their virial radius.
This is not a trivial fact because the abundance of the most massive satellites is determined by a subtle interplay between the accretion rate of systems of corresponding circular velocity and their disruption by the combined effects of the dynamical friction and tidal stripping [95]. Dynamical friction causes satellites to sink to the center at a rate which depends on the mass and orbital parameters of satellite orbit. Orbital parameters, in turn, depend on the cosmological environment of the accreting host halo and are mediated by the tidal stripping which reduces satellite mass as it sinks, thereby rendering dynamical friction less efficient [96-99]. The fact that the concordance $\Lambda \mathrm{CDM}$ model makes an ab initio prediction that the number of massive satellites that can host luminous dwarfs is comparable to observations can therefore be viewed as a success of the model.

Second, the slopes of both the circular velocity function and the $m_{0.6}$ mass function are different in simulations and observations. This implies that we cannot simply match all of the luminous satellites to the subhalos with the largest $V_{\max }$ and $m_{0.6}$, as was sometimes advocated $[89,91]$. The $m_{0.6}$ mass function comparison, in particular, indicates that there should be some subhalos with the $m_{0.6} \sim 10^{7} M_{\odot}$ that do not host the luminous galaxies, and some that do. As I discuss in Section 4.2, this has a strong implication for the physical interpretation of the difference in terms of galaxy formation scenarios.

In summary, the substructure problem can be stated as the discrepancy in the slopes of the circular velocity and $m_{0.6}$ mass functions inferred for observed satellites of the Milky Way and the slopes of these functions predicted for dark matter subhalos in the MW-sized host halos formed in the concordance $\Lambda \mathrm{CDM}$ cosmology.

I believe that stated this way the problem is well defined. Defining the problem in terms of the difference in the actual number of satellites and subhalos is confusing at best, as both numbers are fairly strong functions of subhalo mass or stellar luminosity. Thus, for example, even though the discovery of the ultra-faint dwarfs implies the possible existence of hundreds of them in the halo of the Milky Way $[49,75]$ (this fact has been used to argue that the substructure problem has been "alleviated"), the most recent simulations show that more than 100000 subhalos of mass $m_{\text {sub }}>10^{5} M_{\odot}$ should exist in the Milky Way [31, 32]. (Indeed, it is not obvious a priori that subhalos of mass $10^{5}-10^{6} M_{\odot}$ are too small to host luminous stellar systems of stellar mass $M_{*} \sim 10^{3}-10^{4} M_{\odot}[100]$ 一the stellar masses corresponding to the luminosities of the faintest recently discovered dwarfs. After all, the halos of this mass are expected to be hosting formation of the very first stars [101].) The substructure problem stated in the actual numbers of satellites is therefore alive and well and has not been alleviated in the least.

I would like to close this section by a brief discussion of the comparison of spatial distribution of observed satellites and subhalos. As I noted above, the radial distribution of the observed satellites of the Milky Way is more compact than the radial distribution of subhalos selected using their present day mass or circular velocity $[39,57,102]$. In addition, the observed satellites are distributed in a quite flattened 
structure with its plane almost perpendicular to the disk of the Milky Way [58-62]. Although the spatial distribution of all subhalos is expected to be anisotropic, reflecting the anisotropy of their accretion directions along filaments [44, 45] and, possibly, the fact that some of the satellites could have been accreted as part of the same group of galaxies [103], the expected anisotropy is not as strong as that of the observed Milky Way satellites.

Thus, both the radial distribution and anisotropy of the observed satellites do not match the overall distribution of subhalos in CDM halos. This may be yet another side of the same substructure problem coin and the overall spatial distribution of observed satellites needs to be explained together with the differences in the circular velocity function. (Although distribution of satellites around other galaxies is not mapped as accurately as around the Milky Way, there is evidence for similar planar anisotropy around M31 [60]. The evidence for such anisotropy in other disk galaxies is weak at best [104], although measurements for distant galaxies are more difficult as they require very careful handling of projection effects [105].) I will review a few possible explanations for the substructure problem and differences in the spatial distribution in the following section.

\section{Possible Solutions}

4.1. Modifications to the CDM Model. One possible way to account for the differences of the predicted and observed circular velocity and $m_{0.6}$ mass functions is to assume that $\Lambda C D M$ model is incorrect on the small scales probed by the dwarf galactic satellites. Indeed, the abundance of satellites is sensitive to the amplitude of the power spectrum on the scales corresponding to the total mass of their host halos. For a halo of mass $M$ the comoving scale of fluctuations that seed their formation is

$$
\begin{aligned}
d & =2 R=2\left(\frac{3 M}{4 \pi \Omega_{\mathrm{m} 0} \rho_{\text {crit } 0}}\right)^{1 / 3} \\
& =360.4 \mathrm{kpc}\left(\frac{M}{10^{9} M_{\odot}} \frac{0.3}{\Omega_{\mathrm{m}}}\right)^{1 / 3}\left(\frac{H_{0}}{70}\right)^{-2 / 3},
\end{aligned}
$$

where $\Omega_{\mathrm{m}}$ is the present-day total matter density in units of the present-day critical density, $\rho_{\text {crit } 0} \equiv 3 H_{0}^{2} / 8 \pi G$, and $H_{0}$ is the current Hubble constant in units of $\mathrm{km} / \mathrm{s} / \mathrm{Mpc}$.

If the amplitude of density fluctuations on such scales is considerably suppressed compared to the concordance $\Lambda C D M$ model used in most simulations, the abundance of subhalos can then also be suppressed. Such suppression can be achieved either by suitably varying parameters controlling the amplitude of the small-scale power spectrum within the $\Lambda$ CDM model itself [36], such as the overall normalization of the power spectrum or its large-scale tilt, or by switching to models in which the amplitude at small scales is suppressed, such as the warm dark matter (WDM) structure formation scenarios [36, 106-109]. In these models the abundance of satellites is suppressed both because fewer halos of dwarf mass form in the first place (due to smaller initial amplitude of fluctuations) and because halos that do form have a less-concentrated internal mass distribution, which makes them more susceptible to tidal disruption after they accrete onto their host halo. Models in which dark matter was assumed to be self-interacting, a property that can lead to DM evaporation, have also been proposed and discussed $[110,111]$, but these models both run into a contradiction with other observational properties of galaxies and clusters [112-115] and are now strongly disfavored by observational evidence indicating that dark matter self-interaction is weak [116].

The problem, however, is more subtle than simply suppressing the number of satellites. As discussed above, differences exist between observed and predicted slopes of the circular velocity and mass. The slope is controlled by the slope of the primordial fluctuation spectrum around the scale corresponding to the masses of satellite halos and structural properties of the forming halos. It has not yet been demonstrated convincingly whether both the circular velocity and the $m_{0.6}$ mass functions can be reproduced in any of the models alternative to CDM. In fact, recent measurements of mass distribution in the central regions of observed satellites put stringent constraints on the phase space density and "warmness" of dark matter [75]. At the same time, measurements of the small-scale density power spectrum of the Lyman $\alpha$ forest indicate that fluctuations with the amplitude expected in the $\Lambda$ CDM model at the scales that control the abundance of dwarf mass halos are indeed present in the primordial spectrum $[117,118]$.

While the inner density distribution in observed satellites may still be affected by dark matter warmness in the allowed range of parameter space [77] (see, however, [79]), the models with such parameters would not suppress the overall abundance of satellites considerably. In fact, observations of flux ratios in the multiple image radio lenses appear to require an amount of substructure which is even larger than what is typically found in the CDM halos [119-121], disfavoring models with strongly suppressed abundance of small mass subhalos.

There is thus no compelling reason yet to think that the observed properties of galactic satellite populations are more naturally reproduced in these models. In the subsequent discussion, I will therefore use Occam's razor and focus on the possible explanations of the differences between observed satellites and subhalos in simulations within the $\Lambda \mathrm{CDM}$ model. The prime suspect in producing the discrepancy is the still quite uncertain physics of galaxy formation. After all, a similar problem exists for objects of larger masses and luminosities if we compare the slope of the luminosity function and the halo mass function [122-125] or the predicted and observed abundance of galaxies in the nearby low density "field" regions [126].

4.2. Physics of Galaxy Formation. Several plausible physical processes can suppress gas accretion and star formation in dwarf dark matter halos. The cosmological UV background, which reionized the universe at $z \gtrsim 6$, heats the intergalactic gas and establishes a characteristic time-dependent minimum mass for halos that can accrete gas [127-134]. 
The gas in the low-mass halos may be photoevaporated after reionization [135-137] or blown away by the first generation of supernovae [138-141] (see [142]). At the same time, the ionizing radiation may quickly dissociate molecular hydrogen, the only efficient coolant for lowmetallicity gas in such halos, and prevent star formation even before the gas is completely removed [143]. Even if the molecular hydrogen is not dissociated, cooling rate in halos with virial temperature $T_{\text {vir }} \lesssim 10^{4} \mathrm{~K}$ is considerably lower than in more massive halos [144] and we can therefore expect the formation of dense gaseous disks and star formation to be suppressed in such halos. (The virial virial temperature $T_{\text {vir }}$ is related to the virial mass by $k T_{\text {vir }}=$ $(1 / 2) \mu m_{p} G M_{\mathrm{vir}} / R_{\mathrm{vir}}$, where isothermal temperature profile is assumed for simplicity. The virial mass and radius are related by definition as $M_{\mathrm{vir}}=4 \pi / 3 \times \Delta_{\mathrm{vir}} \bar{\rho} R_{\mathrm{vir}}^{3}$. Assuming $\Delta_{\text {vir }}=178$ appropriate for $z \gtrsim 1$ regardless of $\Omega_{0}$, this gives $M_{\text {vir }} \approx 5.63 \times 10^{7} h^{-1} M_{\odot}\left(\Omega_{0} / 0.3\right)^{-1 / 2}((1+z) / 11)^{-3 / 2}\left(T_{\text {vir }} /\right.$ $\left.10^{4} \mathrm{~K}\right)^{3 / 2}$.) Another potential galaxy formation suppression mechanism is the gas stripping effect of shocks from galactic outflows and cosmic accretion [145, 146]. Finally, even if the gas is accreted and cools in small-mass halos, it is not guaranteed that it will form stars if gas does not reach metallicities and surface densities sufficient for efficient formation of molecular gas and subsequent star formation $[39,147-150]$.

The combined effect of these processes is likely to leave most of dark matter halos with masses $\lesssim$ few $\times 10^{9} M_{\odot}$ dark, and could have imprinted a distinct signature on the properties of the dwarf galaxies that did manage to form stars before reionization. In fact, if all these suppressing effects are as efficient as is usually thought, it is quite remarkable that galaxies such as the recently discovered ultra-faint dwarfs exist at all. One possibility extensively discussed in literature is that they managed to accrete a certain amount of gas and form stars before the universe was reionized [81-84, 87, 151153]. Direct cosmological simulations do show that dwarf galaxies forming at $z>6$ bear striking resemblance to the faint dwarf spheroidal galaxies orbiting the Milky Way [154-156] and their predicted abundance around the Milky Way is consistent with estimates of the abundance of the faintest satellites [151]. Alternatively, some authors argued $[78,89,91,157]$ that observed dwarf satellite galaxies could be in much more massive subhalos than was indicated by simple estimates of dynamical masses and circular velocities using stellar velocity dispersions. In this case, the relatively large halo mass could allow an object to resist the suppressing effects of the UV background.

Cosmological simulations also clearly show that the subhalos found within virial radii of larger halos at $z=0$ have on average lost significant amount of mass and have been considerably more massive in the past $[22,38,39]$. The dramatic loss of mass occurs due to the tidal forces that subhalos experience as they orbit in the potential of the host. A significant fraction of the luminous dwarf satellites therefore can be associated with those subhalos that have been substantially more massive in the past and hence more resilient against galaxy formation suppressing processes.
Such subhalos could have had a window of opportunity to form their stellar systems even if the subhalos they are embedded in today have relatively small mass [39].

4.3. Models for Luminous Satellite Population. Given the galaxy formation suppression mechanisms and evolutionary scenarios listed above, the models aiming to explain the substructure problem can be split into the following broad classes: (1) the "threshold galaxy formation models" in which luminous satellites are embedded in the most massive subhalos of CDM halos and their relatively small number indicates the suppression of galaxy formation in subhalos of circular velocity smaller than some threshold value [78, 89,157 ] and (2) "selective galaxy formation models" in which only a fraction of small subhalos of a given current $V_{\max }$ and mass host luminous satellites while the rest remain dark.

In the second class of models the processes determining whether a subhalo hosts a luminous galaxy can be the reionization epoch $[81,87,152-154,158]$ : subhalos that assemble before the intergalactic medium was heated by ionized radiation become luminous. The observed faint dwarfs can then be the "fossils" of the pre-reionization epoch $[154,155]$. Subhalo may also form a stellar system if its mass assembly history was favorable for galaxy formation [39]: namely, luminous subhalos are those that have had sufficiently large mass during a period of their evolution to allow them to overcome the star formation suppression processes.

Several models using a combination of the processes and scenarios outlined above have been shown to reproduce the gross properties of observed population of satellites reasonably well $[39,84,85,87]$. How can we test different classes of models and differentiate between specific ones?

First, I think the fact the $m_{0.6}$ mass function for observed satellites has a different slope compared to simulation predictions (Figure 8) favors the second class of the selective galaxy formation models, at least for the brighter "classical" satellites. Indeed, given what we know about the average mass loss of subhalos, it is more natural to associate the observed systems with the halos of the largest mass prior to accretion [39] rather than with the subhalos with the largest current masses. Second, the predicted number of the weakly evolving pre-reionization objects $[84,151,153]$, the extended star formation histories of most of the observed dwarf satellites $[53-55,159]$ (in fact, in terms of star formation the main difference between the dIrr and dSph galaxies appears to be presence or lack of star formation in the last 2 billion years before $z=0[54]$ ), and the significant spread in metallicities and certain isotope ratios [160] indicate that majority of "classical" dwarfs have not formed most of their stars before reionization but have formed their stars over rather extended period of time $(\sim 10 \mathrm{Gyr})$. It is still possible, however, that a sizable fraction of the ultra-faint dwarfs are the "pre-reionization fossils" [151, 155, 161, 162], if star formation efficiency in these objects is greatly suppressed [84] compared to that of brighter dwarfs. 
Interesting additional clues and constraints on the galaxy formation models available for the dwarf satellites of the Milky Way are the measurements of the total dynamical mass within their stellar extent. Observations show that the total masses within a fixed aperture of the observed satellites are remarkably similar despite a several order of magnitude span in dwarf satellite luminosities [49, 78, $88,163,164]$. For example, the range of masses within $0.6 \mathrm{kpc}$ shown in Figure 8 is only an order of magnitude. Furthermore, existence of the tight correlation between total dynamical mass within the half-light radius, $M\left(r_{\text {half }}\right)$, and the corresponding radius $r_{\text {half }}$ [79] for bright $\mathrm{dSph}$ galaxies implies a very similar inner dark matter density profile of their host halos. Recently, Strigari et al. [88] have shown that the mass estimated within the central $300 \mathrm{pc}, m(<0.3 \mathrm{kpc}) \equiv$ $m_{0.3}$, for all of the dwarfs with kinematic data varies by at most a factor of four, while the luminosity of the galaxies varies by more than four orders of magnitude.

These observational measurements put constraints on the range of masses of CDM subhalos that can host observed satellites. To estimate this range, we should first note that for the CDM halos described by the NFW profile [18] with concentration $c \equiv R_{\mathrm{vir}} / r_{s}$ (where $r_{s}$ is the scale radius-the radius at which the density profile has logarithmic slope of -2 ) the dependence of the mass within a fixed small radius $x \equiv r / R_{\text {vir }}$ on the total virial mass is

$$
\begin{gathered}
m(<x)=M_{\mathrm{vir}} \frac{f(c x)}{f(c)}, \\
\text { where } f(x) \equiv \ln (1+x)-\frac{x}{1+x},
\end{gathered}
$$

and is quite weak for $r=300$ pc: $m_{0.3} \propto M_{\text {vir }}^{0.3-0.35}$, as shown in Figure 9. Indeed, even for a halo with the Milky Way mass at $z=0$ we expect $m_{0.3} \approx 4 \times 10^{7} M_{\odot}$, a value not too different from those measured for the nearby dwarf spheroidals. Physically, the weak dependence of the central mass on the total mass of the halo reflects the fact that central regions of halos form very early by mergers of small-mass halos. Given that the rms amplitude of density perturbations on small scales is a weak function of scale, the central regions of halos of different mass form at a similar range of redshifts and thus have similar central densities reflecting the density of the universe when the inner region was assembled. At earlier epochs the dependence is stronger because $300 \mathrm{pc}$ represents a larger fraction of the virial radius of halos.

Note that the relation plotted in Figure 9 is for isolated halos unaffected by tidal stripping. Taking into account effects of tidal stripping results in even flatter relation [86]: $m_{0.3} \propto M_{\mathrm{vir}}^{0.25}$, which also has a lower normalization (smaller $m_{0.3}$ for a given $M_{\mathrm{vir}}$. This is likely due to a combination of two effects: (1) the halos of larger mass have lower concentrations and thus can be stripped more efficiently and (2) the halos of larger mass can sink to smaller radii after they accrete and experience relatively more tidal stripping. Overall, the effect of tidal stripping and heating on $m_{0.3}$ appears to be substantial and cannot be neglected.

Finally, Figure 9 shows that the virial mass range corresponding to a given range of $m_{0.3}$ is quite different for

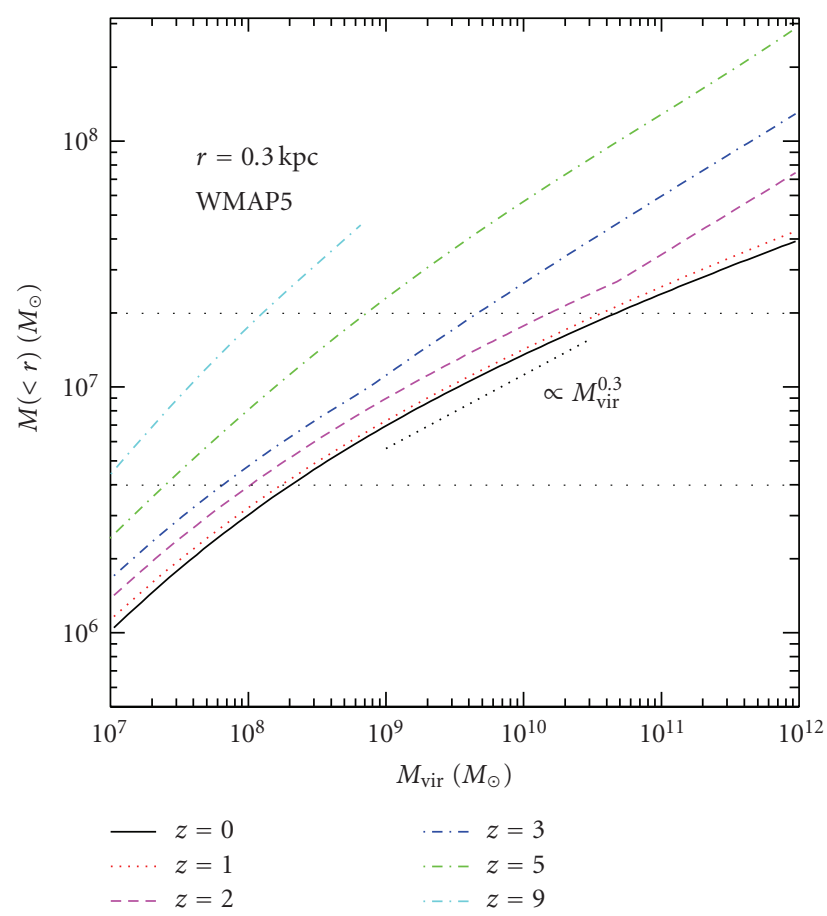

FIGURE 9: The mass within central 300 pc versus the total virial mass of an NFW halo predicted using the concentration mass relation $c\left(M_{\text {vir }}\right)$ at different redshifts in the concordance WMAP 5-year best fit cosmology. The horizontal dotted lines indicate the range of $m_{0.3}$ masses measured for the Milky Way dwarf satellites.

halos that form at $z>2$ compared to those that form at later epochs due to the rapidly evolving NFW concentration concentration for a fixed halo mass [165]. The mass $m_{0.3}$ can therefore be only interpreted in the context of a model for subhalo evolutionary histories.

Several recent studies have used such models to show that the nearly constant central mass of the satellite halos is their natural outcome [84-86]. This outcome can be understood as a combination of the weakness of the $m_{0.3}-$ $M_{\text {vir }}$ correlation and the fact that in the galaxy formation models galaxy luminosity $L$ must be a nonlinear function of $M_{\mathrm{vir}}$ in order to produce a faint-end slope of the galaxy luminosity function much shallower than the slope of the small-mass tail of the halo mass function.

For example, if the faint-end slope of the luminosity function is $\xi$ (i.e., $d n(L) / d L \propto L^{\xi}$ ) and the slope of the halo mass function at small mass end is $\zeta\left(d n(M) / d M \propto M^{\zeta}\right)$ and we assume for simplicity a one-to-one monotonic matching between galaxies and halos $n(>L)=n(>M)$ (see [166168] for the detailed justification for such assumption), the implied slope of the $L-M_{\mathrm{vir}}$ relation is $\beta=(1+\zeta) /(1+\xi)$, which for the fiducial values of $\zeta \approx-2$ and $\xi \approx-1.2$ gives $\beta \approx 5$. In semianalytic models, such a steep nonlinear $L-M_{\mathrm{vir}}$ relation is usually assumed to be set by either suppression of gas accretion due to UV heating or by gas blowout due to SN feedback, e.g., [83]). If I instead assume the faint-end slope of $\xi \approx 1.5-1.6$ as suggested by recent measurement of [125], the relation is shallower but is still nonlinear: $\beta \approx 2$. 


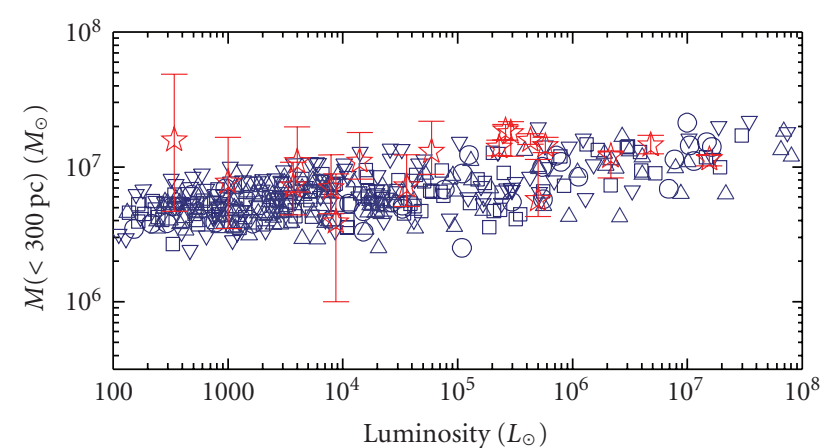

FIGURE 10: The mass within the central 300 pc versus luminosity for the dwarf satellites of the Milky Way (stars with error bars, see [88]). The open symbols of different types show the expected relation for subhalos in three different Milky Way-sized halos formed in the simulations of the concordance $\Lambda$ CDM cosmology if the luminosity of the subhalos is related to their virial mass at accretion epoch as $L=5 \times 10^{3} L_{\odot}\left(M_{\mathrm{vir}, \mathrm{acc}} / 10^{9} M_{\odot}\right)^{2.5}$ (see text for discussion).

Regardless of the specific processes producing the nonlinear luminosity-mass relation, for a relation of the form $L \propto M_{\mathrm{vir}}^{\beta}$, we have $L \propto m_{0.3}^{\gamma}$, where $\gamma \approx \beta / 0.25$, using the $m_{0.3}-M_{\text {vir }}$ relation above taking into account effects of tidal stripping. To account for a smaller than a factor of four spread in central masses for approximately four orders of magnitude spread in luminosity one needs $\gamma \approx 6-8$ or $\beta \approx 2-4$, the values not too different from the estimate above. Thus, the weak correlation of the $m_{0.3}$ and luminosity will be the natural outcome of any CDM-based galaxy formation model which reproduces the slope of the faint end of the galaxy luminosity function.

Within the framework I just described, the slope of the $m_{0.3}-L$ correlation depends on the slope of the $L-M_{\text {vir,acc }}$ correlation $\beta$. The models published so far [84-86], as well as the simple model above with the slope $\beta \approx 2-3$, predict that the slope of the $L-m_{0.3}$ relation is shallow but is nevertheless not zero. Constraining this slope with future observations will tighten constraints on the galaxy formation models and will tell us more about the $L-M_{\text {vir }}$ correlation if such exists.

To illustrate the points just made, Figure 10 shows the $m_{0.3}-L$ relation for the observed nearby dwarfs [88] and subhalos found within $300 h^{-1} \mathrm{kpc}$ around three different MW-sized halos formed in the concordance cosmological model (see $[39,151]$ for simulation details).(The simulations used here do not reliably resolve the mass within 300 pc. Therefore, in order to calculate the mass I have used the mass and concentration of each subhalo at the epoch when it was accreted and computed evolution of their density profile given the mass loss they experienced by the present epoch, as measured in cosmological simulations, and using results of controlled high-resolution simulations of tidal evolution [43], which predict the evolution of the NFW concentration of halos as a function of tidal mass loss. The mass $m_{0.3}$ was then computed from the evolved density profile.) To assign luminosity to a given subhalo I follow the logic of the model presented in [39], which posits that the brightest observed satellites should correspond to the subhalos which have the largest mass before they were accreted. In this model the luminosity of stellar systems should positively correlate with the mass of its host subhalo before it was accreted onto the MW progenitor, $M_{\text {vir,acc }}$ :

$$
L_{V}=5 \times 10^{3} L_{\odot}\left(\frac{M_{\mathrm{vir}, \mathrm{acc}}}{10^{9} M_{\odot}}\right)^{2.5} .
$$

The power law form of the relation is motivated by the approximately power law form of the galaxy luminosity and halo mass functions at faint luminosities and small masses. The actual parameters were chosen such that luminosities of the most massive subhalos roughly match the luminosities of the most massive satellites, such as the SMC and LMC $\left(L \sim 10^{8}-10^{9} L_{\odot}\right.$ ). (Figure 10 does not show the most massive subhalos which would correspond to the systems such as the Large Magellanic Clouds, which have luminosities $L>$ $10^{8} L_{\odot}$ outside the range shown in the figure. This is justified because observational points shown in the figure include only the fainter dwarf spheroidal galaxies.) After all, the first order of business for all the models of satellite population is to reproduce the abundance and luminosities of the most massive $\left(V_{\max } \gtrsim 40 \mathrm{~km} / \mathrm{s}\right.$ ) satellites. The slope of the relation in (4) was set to reproduce the range of observed satellite luminosities and flatness of the $m_{0.3}-L$ relation. Note that this model does not assume any threshold for formation of galaxies. It simply implies that the efficiency with which baryons are converted into stars, $f_{*}=M_{*} / M_{\text {vir }}$, steadily decreases with decreasing $M_{\mathrm{vir}}$ at the rate given by (4). The slope of 2.5 assumed in the equation above is on the lower end of the slopes suggested by matching of the faint end of galaxy luminosity function and small-mass end of halo mass functions discussed above, but is within the current uncertainties in the slope of the former [125]. Assuming such relation for field halos would therefore also reproduce the observed faint end of galaxy luminosity function within current uncertainties.

Figure 10 shows that the model with parameters of (4) is in agreement with observed measurements of the $m_{0.3}-L_{V}$ relation. The results will not change drastically if a somewhat steeper $(\approx 3-3.5)$ slope is assumed. (The model of $(4)$ is similar to the model $1 \mathrm{~B}$ in the recent study by Koposov et al. [84], which assumes that stellar mass scales as $M_{*}=$ $f_{*} M_{0}\left(M_{\mathrm{vir}, \mathrm{acc}} / M_{0}\right)^{1+\alpha}$, These authors find that the model reproduces the luminosity function of satellites for $f_{*} \approx$ $1.7 \times 10^{-4}, M_{0}=10^{10} M_{\odot}$, and $\alpha=2$, which gives $M_{*}=$ $1.7 \times 10^{3}\left(M_{\mathrm{sat}} / 10^{9} M_{\odot}\right)^{3}$, quite similar to the relation given by (4). Koposov et al. adjust parameters to match the observed luminosity function and then show that $L-m_{0.3}$ relation is reproduced, while I adopted the opposite route here. The key difference between the models is that their model assumes a ceiling on the value of $M_{*} / M_{\text {vir,acc }}$, while I assume no such ceiling. Absence of the ceiling on star formation efficiency is actually important for bright satellites (see Figure 14).)

Having fixed the parameters of the $L-M_{\text {vir,acc }}$ relation, we can then ask the question of whether the luminosity function of satellites would be reproduced self-consistently by such a model. We can use the observed luminosity functions corrected for completeness for the faintest dwarfs 


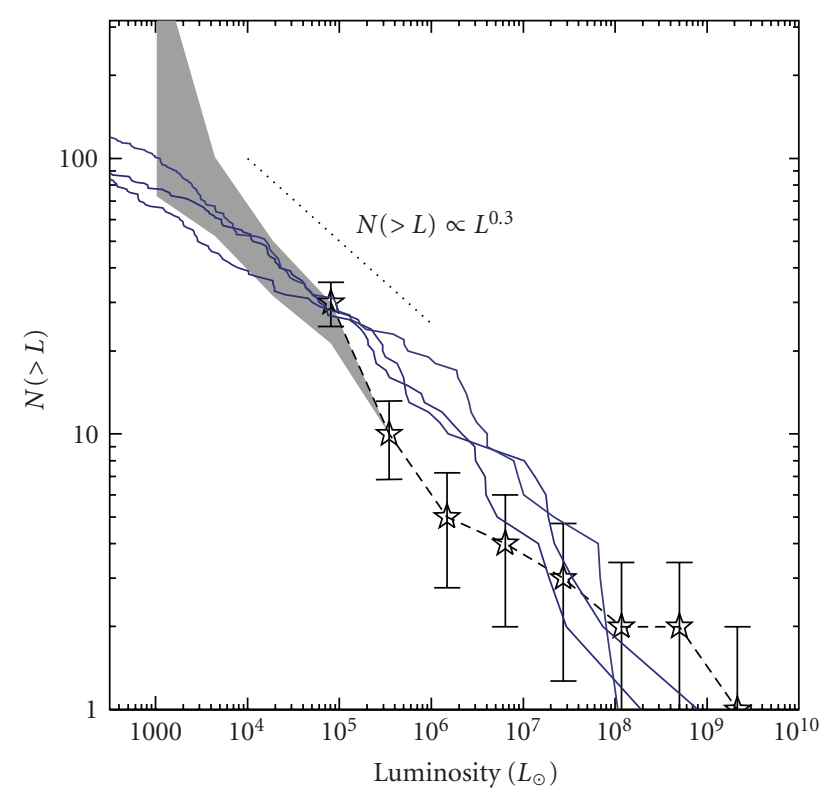

FIgURE 11: The cumulative luminosity function of subhalos in the three MW-sized halos shown in Figure 10 with the simple luminosity assignment ansatz of (4). The luminosity function includes all subhalos within $417 \mathrm{kpc}$ from the center of each halo, the same radius as was used to construct the luminosity function of the observed satellites shown in Figure 6. The points with (Poisson) error bars, connected by the dashed line, and the shaded region show the observed and inferred satellite luminosity functions within the same radius for classical and ultra-faint dwarfs, respectively, as compiled by [56] (see text for details).

from [56] (shown in Figure 6) to test this. Figure 11 shows the subhalo luminosity functions constructed using subhalos identified within $417 \mathrm{kpc}$ (the same outer radius used in the construction of the observed luminosity function by [56]) in the simulations and the simple luminosity assignment scheme of (4). The points with (Poisson) error bars and the shaded region show the observed luminosity functions for the classical satellites and for ultra-faint dwarfs, as compiled by [56]. As I noted before, the current samples of the ultrafaint satellites exist only over a fraction of the sky and there are reasons to expect that they are incomplete for distances larger than $\approx 50 \mathrm{kpc}$. The shaded region, therefore, represents the likely bracket of the possibilities. The lower edge of the region is the luminosity function in the case when the observed luminosity function of the ultra-faints is simply corrected for the fractional sky coverage. The upper edge of the shaded region shows a combined correction for both the sky coverage and radial distribution assuming that ultrafaints have the same radial distribution as mass-selected subhalos in the Via Lactea I simulation [56].

Figure 11 shows that although the model and observed luminosity functions do not match perfectly, they are in reasonable agreement. One should remember a number of uncertainties in the model luminosity functions. First, the masses of host halos can be a factor of two or so larger than the mass of the Milky Way and the cosmology of the simulations may not be exactly correct. Second, the $L-M_{\text {acc }}$

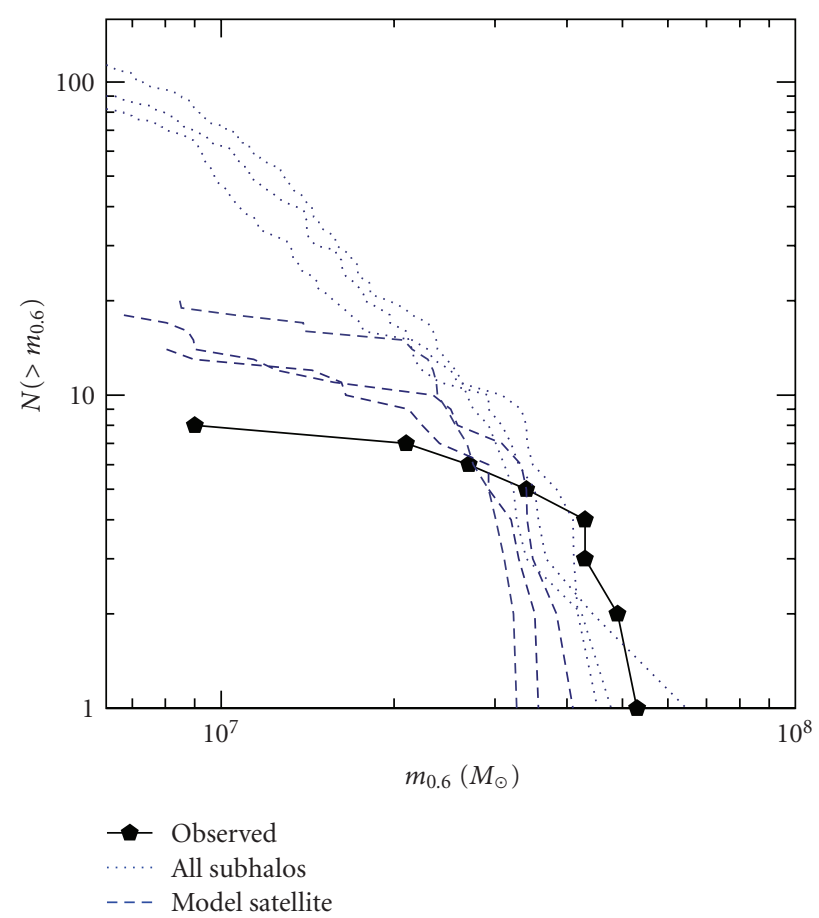

Figure 12: The cumulative $m_{0.6}$ function of subhalos in the $\Lambda \mathrm{CDM}$ simulations of three MW halos (dotted lines) and for the observed dSph Milky Way satellites (points, connected by solid line) Sagittarius was excluded due to its very large mass errors), as measured by [90]. The dashed lines show the mass function for subhalos with $L \geq 2.6 \times 10^{5} L_{\odot}$ and $d<270 \mathrm{kpc}$ (the same range of luminosities and distances as for the observed satellites) with luminosities assigned using (4). I have excluded the two most luminous objects from the model luminous satellites to account for the fact that SMC and LMC are not included in the observational sample and one additional object to account for noninclusion of the Sagittarius dwarf in the comparison.

relation assumed in the model can be somewhat different from that assumed in the figure: for example, the slope can be somewhat steeper and normalization lower, which would make both the $m_{0.3}-L$ relation flatter and the luminosity functions closer to observations. The fiducial relation in (4) was chosen to have the shallowest slope (2.5) still reasonably consistent with observations.

Figure 12 shows comparison of the $m_{0.6}$ mass functions for the observed "classical" dwarf spheroidal satellites of the Milky Way [90] and predicted mass function for the entire subhalo population of the MW-sized halos within $270 \mathrm{kpc}$ (the largest distance of the observed satellite included in the comparison) and the subhalos with $L>2.6 \times 10^{5} L_{\odot}$ (the smallest dSph luminosity included in the observed sample) with luminosities assigned using (4). I have excluded the Sagittarius dwarf from this comparison as its $m_{0.6}$ mass has very large errors [90]. The number of the predicted luminous satellites was reduced by three to account for exclusion of the Sagittarius, SMC, and LMC from the comparison. The figure shows that the model predicts the range of $m_{0.6}$ quite similar to that measured for the observed luminous dSphs. The shape of the mass function is also in reasonably good 
agreement with the data. Although there are somewhat more predicted satellites at small masses, this is likely due to the somewhat larger virial mass of the simulated halos $(\approx$ $2-3 \times 10^{12} M_{\odot}$ ) compared to the mass of the Milky Way $\left(\approx 10^{12} M_{\odot}\right)$. We expect the number of subhalos to scale approximately linearly with host mass and so the difference in the virial mass of the Milky Way and simulated halos can account for the difference with observations in Figure 12. There is some discrepancy at the largest $m_{0.6}$ values, but it is not clear just how significant the discrepancy is given that the typical errors on the $m_{0.6}$ measurements for these galaxies are $\approx 20 \%-40 \%$.

Finally, Figure 13 compares cumulative radial distribution of the observed "classical" Milky Way satellites within $280 \mathrm{kpc}$ and satellites with similar luminosities and within the same distance from their host halo in the model of (4). The figure also shows the cumulative distribution of all subhalos selected using their current $V_{\max }$. The predicted distribution of bright luminous satellites is somewhat more radially concentrated than the distribution of the $V_{\max }$-selected subhalos and is in reasonable agreement with the observed distribution both in its median and in the overall shape.

Thus, the observed $m_{0.3}-L$ relation, the luminosity function, the $m_{0.6}$ mass function, and the radial distribution of the observed satellites can all be reproduced simultaneously with such a simple dwarf galaxy formation scenario. The observational uncertainties in these statistics are still quite large, which leaves significant freedom in the parameters of (4) and in its functional form. (It is quite possible that relation between luminosity and mass is more complicated than (4). For example, the normalization of the $L-M_{\text {viracc }}$ relation can evolve with redshift because luminosity may be determined both by the mass of the halo at the accretion epoch and by the period of time before its accretion during which it was sufficiently massive to withstand star formation suppressing processes. Such redshift dependence would be an extra parameter which would generate scatter in the $L-M_{\text {vir,acc }}$ relation.) It is also possible that all of these statistics may be reproduced in a drastically different scenario. Nevertheless, the success of such simple model is encouraging and it is interesting to discuss its potential implications.

First of all, (4) implies that all of the observed Milky Way dSph satellites had virial masses $M_{\text {vir,acc }} \gtrsim 5 \times 10^{8} M_{\odot}$ when they were accreted and these masses may span the range up to $\sim 5 \times 10^{10} M_{\odot}$ (the actual range depends sensitively on the slope of the $L-M_{\text {viracc }}$ relation). This shows that progenitors of the observed satellites could have had a wide range of virial masses, even though the range of their $m_{0.3}$ and $m_{0.6}$ masses is narrow.

An interesting implication of the value of lowest mass of the range of masses above is that whatever gas the small-mass halos $\left(M_{\text {vir }} \lesssim 5 \times 10^{8} M_{\odot}\right)$ are able to accrete, it should remain largely unused for star formation, and of course would not be blown away by supernovae (given that the model implies that such objects should have no stars or supernovae). If some of this gas is neutral, it can contribute to HI absorption lines in the spectra of quasars and distant galaxies. If this gas is enriched, it can also produce absorption lines of heavier

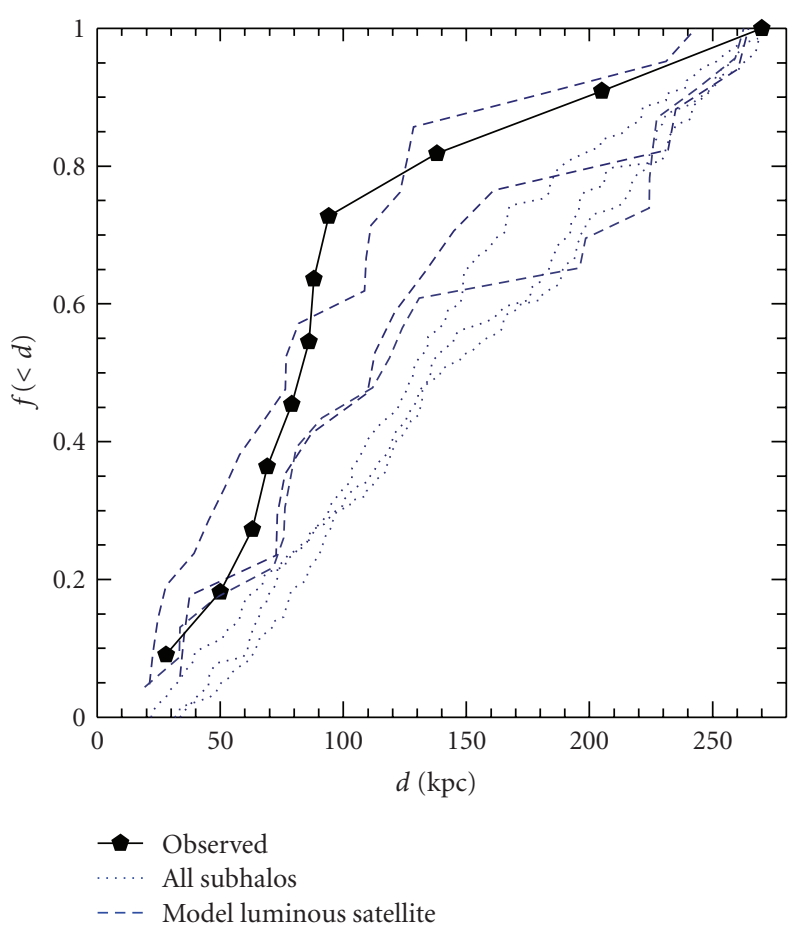

FIGURE 13: The cumulative radial distribution of the observed "classical" Milky Way satellites (solid points connected by the solid line) within $280 \mathrm{kpc}$ and satellites with similar luminosities and within the same distance from their host halo in the model of (4) (dashed lines). The figure also shows the cumulative distribution of all subhalos selected using their current $V_{\max }$ (dotted lines).

elements. At lower redshifts, the neutral gas in the otherwise starless or very faint halos could manifest itself in the form of the High Velocity Clouds (HVCs) abundant in the Local Group [169-172] and around other galaxies.

Second, as I noted above the slope of the $L-M_{\text {vir }}$ relation required to explain the weak dependence of $m_{0.3}$ on luminosity is not surprising, given what we know about the faint-end slope of galaxy luminosity function and what we expect about the slope of the mass function of their host halos in CDM scenario [173]. The implied normalization of the $L-M_{\text {vir }}$ relation, however, is quite interesting. For example, it indicates that halo of $M_{\text {vir,acc }}=10^{10} M_{\odot}$ should have luminosity of $L_{V}=1.6 \times 10^{6} L_{\odot}$. Converting it to stellar mass assuming $M_{*} / L_{V}=1$ (appropriate for old populations, e.g., [174]) gives $M_{*}=1.6 \times 10^{6} M_{\odot}$. Results of cosmological simulations with UV heating of gas show that halos of $M$ $10^{10} M_{\odot}$ should have been able to accrete almost all of their universal share of baryons, $M_{b}=\left(\Omega_{b} / \Omega_{m}\right) M_{\mathrm{vir}, \mathrm{acc}} \approx 1.7 \times$ $10^{9} M_{\odot}$ (assuming $\Omega_{b} / \Omega_{m} \approx 0.17$ suggested by the WMAP measurements [10]), even in the presence of realistic UV heating $[133,134]$. The derived stellar mass thus implies that only $F_{*} \equiv M_{*} / M_{\text {viracc }} \times\left(\Omega_{m} / \Omega_{b}\right) \approx 0.001$ (i.e., $0.1 \%$ ) of the expected baryon mass was converted into stars in such objects. Such small efficiency $F_{*}$ for systems accretion onto which is not suppressed by the UV heating implies that star formation is dramatically suppressed by some other mechanism. 


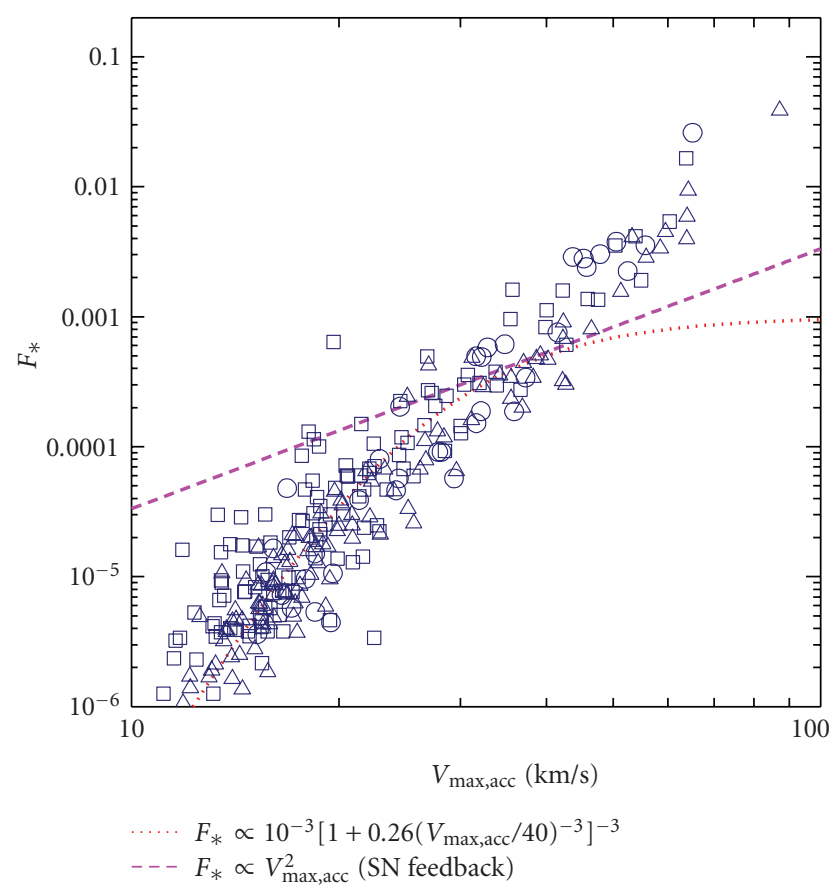

Figure 14: The efficiency of gas conversion into stars, defined as the fraction of baryon mass, expected if the halo accreted its universal fraction of baryons, converted into stars: $F_{*}=$ $\left(\Omega_{m} / \Omega_{b}\right)\left(M_{*} / M_{\text {viracc }}\right)$. The points (as before different symbols correspond to subhalos in three different simulated host halos) show the dependence of $F_{*}$ on the maximum circular velocity of each subhalo at accretion according to luminosity assignment model of (4). The short-dashed line shows the functional form expected if $F_{*}$ was controlled by the suppression of gas accretion due to UV heating of intergalactic gas (model 3B of [84]), while long-dashed line shows scaling expected in efficiency was set by supernova feedback (e.g., [140]).

In fact, the implied efficiency of baryon conversion into the stars, $F_{*}$, in this model is a steep, power law function of mass and circular velocity $\left(F_{*} \approx V_{\text {max,acc }}^{8 \div 9}\right)$, as shown in Figure 14. The figure also shows the functional form one would have expected if the dependence of the efficiency $F_{*}$ on circular velocity was determined by the fraction of gas halos are able to accrete in the presence of the UV radiation. This functional form is almost identical to the fiducial model 3B of Koposov et al. [84] (I used slightly larger value for critical velocity because I use $V_{\max }$ rather than the virial circular velocity used by these authors). The two models have similar behavior at $V_{\text {max,acc }} \lesssim 30 \mathrm{~km} / \mathrm{s}$, but the UV heating model asymptotes to a fixed value of $F_{*}=10^{-3}$ for more massive systems. This model would therefore underpredict luminosities of the most massive satellites in MW-sized halos, which was noticed by Koposov et al. in its failure to reproduce the bright end of the satellite luminosity function. Moreover, the estimates of the efficiency $F_{*}$ for more luminous galaxies, such as the Milky Way, are in the range $F_{*} \sim 0.05-0.2$ [175-178], which would lie roughly on the continuation of the relation in Figure 14 to larger circular velocities shown by symbols. (The $F_{*}-V_{\max }$ relation could plausibly flatten at larger $V_{\max }$, as expected from the halo modeling of the galaxy population, e.g., [173].)

These considerations indicate a very interesting possibility that while the UV heating can mediate accretion of gas into very small mass halos, the efficiency with which the accreted gas is converted into stars in the known luminous galaxies is dramatically suppressed by some other massdependent mechanism. This strong suppression operates not only for halos in which gas accretion is suppressed but for halos of larger masses as well. Note that this suppression mechanism is unlikely to be due to blowout of gas by supernovae, which is expected to give $F_{*} \propto V_{\max }^{2}$ (e.g., [140]), a much shallower relation than the scaling in Figure 14.

While discussion of the nature of this suppression mechanism is outside the scope of this paper, the rapidly improving observational data on the satellite population should shed light on the possible mechanisms.

The exercise presented in this section illustrates just how powerful the combination of luminosity function and radial distribution of satellites, high-quality resolved kinematics data and inferred dynamical constraints on the total mass profile, measurements of star formation histories and enrichment histories can be in understanding formation of dwarf galaxies. The rapidly improving constraints on the mass profiles of the dSph galaxies down to the smallest luminosities $[79,88]$ should further constrain the range of subhalo masses hosting the observed satellites and, by inference, the efficiency of star formation in such small halos. The hints that star formation efficiency is actually a monotonic function of halo mass from galaxies such as the Milky Way to the faintest known galaxies, such as Segue 1 , indicates that the physics learned from the "near-field cosmology" studies of the nearest dwarfs can potentially give us important insights into formation of more massive galaxies as well.

\section{Acknowledgments}

The author would like to thank Brant Robertson, Anatoly Klypin, Nick Gnedin, Jorge Peñarrubia, Erik Tollerud, and James Bullock for useful comments on the manuscript and stimulating discussions on the topics related to the subject of this paper. This work was partially funded by the NSF grants AST-0507666, and AST-0708154. The research was also partially supported by the Kavli Institute for Cosmological Physics at the University of Chicago through grant NSF PHY0551142 and an endowment from the Kavli Foundation. $\mathrm{He}$ also would like to thank Kavli Institute for Theoretical Physics (KITP) in Santa Barbara and organizers of the 2008 KITP workshop "Back to the Galaxy II" where some of the work presented here was carried out, for hospitality and wonderful atmosphere. He has made extensive use of the NASA Astrophysics Data System and arXiv.org preprint server during writing of this paper. 


\section{References}

[1] S. D. M. White and M. J. Rees, "Core condensation in heavy halos-a two-stage theory for galaxy formation and clustering," Monthly Notices of the Royal Astronomical Society, vol. 183, pp. 341-358, 1978.

[2] G. R. Blumenthal, S. M. Faber, J. R. Primack, and M. J. Rees, "Formation of galaxies and large-scale structure with cold dark matter," Nature, vol. 311, no. 5986, pp. 517-525, 1984.

[3] A. Loeb and M. Zaldarriaga, "Small-scale power spectrum of cold dark matter," Physical Review D, vol. 71, no. 10, Article ID 103520, 2005.

[4] S. D. M. White, "Formation and evolution of galaxies," in Cosmology and Large Scale Structure, R. Schaeffer, J. Silk, M. Spiro, and J. Zinn-Justin, Eds., pp. 349-430, Elsevier Scientific, Amsterdam, The Netherlands, 1996.

[5] B. Moore, S. Ghigna, F. Governato, G. Lake, T. Quinn, and J. Stadel, "The structure of cold dark matter halos," in Large Scale Structure: Tracks and Traces, V. Mueller, S. Gottloeber, J. P. Muecket, and J. Wambsganss, Eds., pp. 37-40, World Scientific, Singapore, 1998.

[6] S. Ghigna, B. Moore, F. Governato, G. Lake, T. Quinn, and J. Stadel, "Dark matter haloes within clusters," Monthly Notices of the Royal Astronomical Society, vol. 300, no. 1, pp. 146-162, 1998.

[7] G. Tormen, A. Diaferio, and D. Syer, "Survival of substructure within dark matter haloes," Monthly Notices of the Royal Astronomical Society, vol. 299, no. 3, pp. 728-742, 1998.

[8] B. Moore, S. Ghigna, F. Governato, et al., "Dark matter substructure within galactic halos," The Astrophysical Journal, vol. 524, no. 1, pp. L19-L22, 1999.

[9] A. Klypin, S. Gottlöber, A. V. Kravtsov, and A. M. Khokhlov, "Galaxies in N-body simulations: overcoming the overmerging problem," The Astrophysical Journal, vol. 516, no. 2, pp. 530-551, 1999.

[10] J. Dunkley, E. Komatsu, M. R. Nolta, et al., "Five-year wilkinson microwave anisotropy probe observations: likelihoods and parameters from the WMAP data," The Astrophysical Journal, vol. 180, no. 2, pp. 306-329, 2009.

[11] A. Kravtsov, "Modeling galaxy clustering with cosmological simulations," in Proceedings of the 41st Rencontres de Moriond, 2006.

[12] Y. Birnboim and A. Dekel, "Virial shocks in galactic haloes?" Monthly Notices of the Royal Astronomical Society, vol. 345, no. 1, pp. 349-364, 2003.

[13] D. Kereš, N. Katz, D. H. Weinberg, and R. Davé, "How do galaxies get their gas?" Monthly Notices of the Royal Astronomical Society, vol. 363, no. 1, pp. 2-28, 2005.

[14] W. J. G. de Blok, F. Walter, E. Brinks, C. Trachternach, and R. C. Kennicutt, "High-resolution rotation curves and galaxy mass models from things," The Astronomical Journal, vol. 136, no. 6, pp. 2648-2719, 2008.

[15] W. J. G. de Blok, “The Core-Cusp problem," Advances in Astronomy, 2009.

[16] A. A. Dutton, F. C. van den Bosch, A. Dekel, and S. Courteau, "A revised model for the formation of disk galaxies: low spin and dark halo expansion," The Astrophysical Journal, vol. 654, no. 1, pp. 27-52, 2007.

[17] L. Mayer, F. Governato, and T. Kaufmann, "The formation of disk galaxies in computer simulations," Advanced Science Letters, vol. 1, no. 1, pp. 7-27, 2008.

[18] J. F. Navarro, C. S. Frenk, and S. D. M. White, "A universal density profile from hierarchical clustering," The Astrophysical Journal, vol. 490, no. 2, pp. 493-508, 1997.
[19] A. V. Kravtsov, A. A. Berlind, R. H. Wechsler, et al., "The dark side of the halo occupation distribution," The Astrophysical Journal, vol. 609, no. 1, pp. 35-49, 2004.

[20] L. Gao, S. D. M. White, A. Jenkins, F. Stoehr, and V. Springel, "The subhalo populations of $\Lambda \mathrm{CDM}$ dark haloes," Monthly Notices of the Royal Astronomical Society, vol. 355, no. 3, pp. 819-834, 2004.

[21] F. C. van den Bosch, G. Tormen, and C. Giocoli, "The mass function and average mass-loss rate of dark matter subhaloes," Monthly Notices of the Royal Astronomical Society, vol. 359, no. 3, pp. 1029-1040, 2005.

[22] C. Giocoli, G. Tormen, and F. C. van den Bosch, "The population of dark matter subhaloes: mass functions and average mass-loss rates," Monthly Notices of the Royal Astronomical Society, vol. 386, no. 4, pp. 2135-2144, 2008.

[23] G. Kauffmann, S. D. M. White, and B. Guiderdoni, "The formation and evolution of galaxies within merging dark matter haloes," Monthly Notices of the Royal Astronomical Society, vol. 264, no. 1, pp. 201-218, 1993.

[24] A. Klypin, A. Kravtsov, O. Valenzuela, and F. Prada, "Where are the missing galactic satellites?" The Astrophysical Journal, vol. 522, no. 1, pp. 82-92, 1999.

[25] V. Springel, S. D. M. White, G. Tormen, and G. Kauffmann, "Populating a cluster of galaxies: I. Results at $z=0$," Monthly Notices of the Royal Astronomical Society, vol. 328, no. 3, pp. 726-750, 2001.

[26] J. Diemand, B. Moore, and J. Stadel, "Velocity and spatial biases in cold dark matter subhalo distributions," Monthly Notices of the Royal Astronomical Society, vol. 352, no. 2, pp. 535-546, 2004.

[27] S. P. D. Gill, A. Kneb, and B. K. Gibson, "The evolution of substructure: I. A new identification method," Monthly Notices of the Royal Astronomical Society, vol. 351, no. 2, pp. 399-409, 2004.

[28] J. Weller, J. P. Ostriker, P. Bode, and L. Shaw, "Fast identification of bound structures in large N-body simulations," Monthly Notices of the Royal Astronomical Society, vol. 364, no. 3, pp. 823-832, 2005.

[29] S. R. Knollmann and A. Knebe, "AHF: Amiga's halo finder," The Astrophysical Journal, vol. 182, no. 2, pp. 608-624, 2009.

[30] O. Lahav, P. B. Lilje, J. R. Primack, and M. J. Rees, "Dynamical effects of the cosmological constant," Monthly Notices of the Royal Astronomical Society, vol. 251, pp. 128-136, 1991.

[31] J. Diemand, M. Kuhlen, P. Madau, et al., "Clumps and streams in the local dark matter distribution," Nature, vol. 454, no. 7205, pp. 735-738, 2008.

[32] V. Springel, J. Wang, M. Vogelsberger, et al., "The Aquarius Project: the subhaloes of galactic haloes," Monthly Notices of the Royal Astronomical Society, vol. 391, no. 4, pp. 1685-1711, 2008.

[33] J. Stadel, D. Potter, B. Moore, et al., "Quantifying the heart of darkness with GHALO-a multibillion particle simulation of a galactic halo," Monthly Notices of the Royal Astronomical Society, vol. 398, no. 1, pp. L21-L25, 2009.

[34] A. Jenkins, C. S. Frenk, S. D. M. White, et al., "The mass function of dark matter haloes," Monthly Notices of the Royal Astronomical Society, vol. 321, no. 2, pp. 372-384, 2001.

[35] J. Tinker, A. V. Kravtsov, A. Klypin, et al., "Toward a halo mass function for precision cosmology: the limits of universality," The Astrophysical Journal, vol. 688, no. 2, pp. 709-728, 2008.

[36] A. R. Zentner and J. S. Bullock, "Halo substructure and the power spectrum," The Astrophysical Journal, vol. 598, no. 1, pp. 49-72, 2003. 
[37] J. Diemand, B. Moore, and J. Stadel, "Earth-mass darkmatter haloes as the first structures in the early universe," Nature, vol. 433, no. 7024, pp. 389-391, 2005.

[38] D. Nagai and A. V. Kravtsov, "The radial distribution of galaxies in $\Lambda$ cold dark matter clusters," The Astrophysical Journal, vol. 618, no. 2, pp. 557-568, 2005.

[39] A. V. Kravtsov, O. Y. Gnedin, and A. A. Klypin, "The tumultuous lives of galactic dwarfs and the missing satellites problem," The Astrophysical Journal, vol. 609, no. 2, pp. 482497, 2004.

[40] P. Colín, A. A. Klypin, A. V. Kravtsov, and A. M. Khokhlov, "Evolution of bias in different cosmological models," The Astrophysical Journal, vol. 523, no. 1, pp. 32-53, 1999.

[41] L. Gao, G. de Lucia, S. D. M. White, and A. Jenkins, "Galaxies and subhaloes in ACDM galaxy clusters," Monthly Notices of the Royal Astronomical Society, vol. 352, no. 2, pp. L1-L5, 2004.

[42] A. V. Macciò, B. Moore, J. Stadel, and J. Diemand, "Radial distribution and strong lensing statistics of satellite galaxies and substructure using high-resolution ACDM hydrodynamical simulations," Monthly Notices of the Royal Astronomical Society, vol. 366, no. 4, pp. 1529-1538, 2006.

[43] J. Peñarrubia, J. F. Navarro, and A. W. McConnachie, "The tidal evolution of Local Group dwarf spheroidals," The Astrophysical Journal, vol. 673, no. 1, pp. 226-240, 2008.

[44] A. R. Zentner, A. V. Kravtsov, O. Y. Gnedin, and A. A. Klypin, "The anisotropic distribution of galactic satellites," The Astrophysical Journal, vol. 629, no. 1, pp. 219-232, 2005.

[45] N. I. Libeskind, C. S. Frenk, S. Cole, et al., "The distribution of satellite galaxies: the great pancake," Monthly Notices of the Royal Astronomical Society, vol. 363, no. 1, pp. 146-152, 2005.

[46] X. Kang, F. C. van den Bosch, X. Yang, et al., "The alignment between satellites and central galaxies: theory versus observations," Monthly Notices of the Royal Astronomical Society, vol. 378, no. 4, pp. 1531-1542, 2007.

[47] V. Belokurov, D. B. Zucker, N. W. Evans, et al., "Cats and dogs, hair and a hero: a quintet of new Milky Way companions," The Astrophysical Journal, vol. 654, no. 2, pp. 897-906, 2007.

[48] M. Geha, B. Willman, J. D. Simon, et al., "The least-luminous galaxy: spectroscopy of the Milky Way satellite segue 1," The Astrophysical Journal, vol. 692, no. 2, pp. 1464-1475, 2009.

[49] M. Mateo, "Dwarf galaxies of the Local Group," Annual Review of Astronomy and Astrophysics, vol. 36, no. 1, pp. 435506, 1998.

[50] S. Stierwalt, M. P. Haynes, R. Giovanelli, et al., "The arecibo legacy fast alfa survey. IX. The Leo region H I catalog, group membership, and the H I mass function for the Leo I group," The Astrophysical Journal, vol. 138, no. 2, pp. 338-361, 2009.

[51] J. Woo, S. Courteau, and A. Dekel, "Scaling relations and the fundamental line of the Local Group dwarf galaxies," Monthly Notices of the Royal Astronomical Society, vol. 390, no. 4, pp. 1453-1469, 2008.

[52] E. K. Grebel and J. S. Gallagher III, "The impact of reionization on the stellar populations of nearby dwarf galaxies," The Astrophysical Journal, vol. 610, no. 2, pp. L89L92, 2004.

[53] A. E. Dolphin, D. R. Weisz, E. D. Skillman, and J. A. Holtzman, "Star formation histories of Local Group dwarf galaxies," http://arxiv.org/abs/astroph/0506430.

[54] C. Orban, O. Y. Gnedin, D. R. Weisz, E. D. Skillman, A. E. Dolphin, and J. A. Holtzman, "Delving deeper into the tumultuous lives of galactic dwarfs: modeling star formation histories," The Astrophysical Journal, vol. 686, no. 2, pp. 10301044, 2008.

[55] E. Tolstoy, V. Hill, and M. Tosi, "Star-formation histories, abundances, and kinematics of dwarf galaxies in the Local Group," Annual Review of Astronomy and Astrophysics, vol. 47, pp. 371-425, 2009.

[56] E. J. Tollerud, J. S. Bullock, L. E. Strigari, and B. Willman, "Hundreds of Milky Way satellites? Luminosity bias in the satellite luminosity function," The Astrophysical Journal, vol. 688, no. 1, pp. 277-289, 2008.

[57] B. Willman, F. Governato, J. J. Dalcanton, D. Reed, and T. Quinn, "The observed and predicted spatial distribution of Milky Way satellite galaxies," Monthly Notices of the Royal Astronomical Society, vol. 353, no. 2, pp. 639-646, 2004.

[58] D. Lynden-Bell, "The Fornax-Leo-Sculptor system," The Observatory, vol. 102, pp. 202-208, 1982.

[59] S. R. Majewski, "The Fornax-Leo-Sculptor stream revisited," The Astrophysical Journal, vol. 431, no. 1, pp. L17-L21, 1994.

[60] F. D. A. Hartwick, "The structure of the outer halo of the galaxy and its relationship to nearby large-scale structure," The Astronomical Journal, vol. 119, no. 5, pp. 2248-2253, 2000.

[61] P. Kroupa, C. Theis, and C. M. Boily, "The great disk of Milky-Way satellites and cosmological sub-structures," Astronomy \& Astrophysics, vol. 431, no. 2, pp. 517-521, 2005.

[62] M. Metz, P. Kroupa, and H. Jerjen, "Discs of satellites: the new dwarf spheroidals," Monthly Notices of the Royal Astronomical Society, vol. 394, no. 4, pp. 2223-2228, 2009.

[63] R. A. Ibata, G. Gilmore, and M. J. Irwin, "A dwarf satellite galaxy in Sagittarius," Nature, vol. 370, no. 6486, pp. 194-196, 1994.

[64] D. R. Law, K. V. Johnston, and S. R. Majewski, "A two micron all-sky survey view of the Sagittarius dwarf galaxy. IV. Modeling the Sagittarius tidal tails," The Astrophysical Journal, vol. 619, no. 2, pp. 807-823, 2005.

[65] B. Willman, M. R. Blanton, A. A. West, et al., "A new Milky Way companion: unusual globular cluster or extreme dwarf satellite?" The Astronomical Journal, vol. 129, no. 6, pp. 26922700, 2005.

[66] V. Belokurov, D. B. Zucker, N. W. Evans, et al., "A faint new Milky Way satellite in bootes," The Astrophysical Journal, vol. 647, no. 2, pp. L111-L114, 2006.

[67] D. B. Zucker, V. Belokurov, N. W. Evans, et al., "A curious Milky Way satellite in Ursa Major," The Astrophysical Journal, vol. 650, no. 1, pp. L41-L44, 2006.

[68] D. B. Zucker, V. Belokurov, N. W. Evans, et al., "A new Milky Way dwarf satellite in canes venatici," The Astrophysical Journal, vol. 643, no. 2, pp. L103-L106, 2006.

[69] M. J. Irwin, V. Belokurov, N. W. Evans, et al., "Discovery of an unusual dwarf galaxy in the outskirts of the Milky Way," The Astrophysical Journal, vol. 656, no. 1, pp. L13-L16, 2007.

[70] S. Koposov, J. T. A. de Jong, V. Belokurov, et al., "The discovery of two extremely low luminosity Milky Way globular clusters," The Astrophysical Journal, vol. 669, no. 1, pp. 337-342, 2007.

[71] S. M. Walsh, H. Jerjen, and B. Willman, "A pair of boötes: a new Milky Way satellite," The Astrophysical Journal, vol. 662, no. 2, pp. L83-L86, 2007.

[72] B. Willman, "In pursuit of the least luminous galaxies," Advances in Astronomy, vol. 2010, Article ID 285454, 11 pages, 2010.

[73] E. N. Kirby, J. D. Simon, M. Geha, P. Guhathakurta, and A. Frebel, "Uncovering extremely metal-poor stars in the 
Milky Way's ultrafaint dwarf spheroidal satellite galaxies," The Astrophysical Journal, vol. 685, no. 1, pp. L43-L46, 2008.

[74] S. Koposov, V. Belokurov, N. W. Evans, et al., "The luminosity function of the Milky Way satellites," The Astrophysical Journal, vol. 686, no. 1, pp. 279-291, 2008.

[75] L. E. Strigari, J. S. Bullock, M. Kaplinghat, et al., "A large dark matter core in the Fornax dwarf spheroidal galaxy?" The Astrophysical Journal, vol. 652, no. 1, pp. 306-312, 2006.

[76] J. Klimentowski, E. L. Łokas, S. Kazantzidis, F. Prada, L. Mayer, and G. A. Mamon, "Mass modelling of dwarf spheroidal galaxies: the effect of unbound stars from tidal tails and the Milky Way," Monthly Notices of the Royal Astronomical Society, vol. 378, no. 1, pp. 353-368, 2007.

[77] R. F. G. Wyse and G. Gilmore, "Observed properties of dark matter on small spatial scales," in Proceedings of the International Astronomical Union, J. Davies and M. Disney, Eds., vol. 244 of IAU Symposium, pp. 44-52, Cambridge University Press, 2008.

[78] J. Peñarrubia, A. W. McConnachie, and J. F. Navarro, "The cold dark matter halos of Local Group dwarf spheroidals," The Astrophysical Journal, vol. 672, no. 2, pp. 904-913, 2008.

[79] M. G. Walker, M. Mateo, E. W. Olszewski, J. Peñarrubia, N. W. Evans, and G. Gilmore, "A universal mass profile for dwarf spheroidal galaxies?" The Astrophysical Journal, vol. 704, no. 2, pp. 1274-1287, 2009.

[80] L. E. Strigari, "Kinematics of Milky Way satellites: rotational limits and mass estimation," Advances in Astronomy, 2009.

[81] J. S. Bullock, A. V. Kravtsov, and D. H. Weinberg, "Reionization and the abundance of galactic satellites," The Astrophysical Journal, vol. 539, no. 2, pp. 517-521, 2000.

[82] R. S. Somerville, "Can photoionization squelching resolve the substructure crisis?" The Astrophysical Journal, vol. 572, no. 1, pp. L23-L26, 2002.

[83] A. J. Benson, C. S. Frenk, C. G. Lacey, C. M. Baugh, and S. Cole, "The effects of photoionization on galaxy formation. II. Satellite galaxies in the Local Group," Monthly Notices of the Royal Astronomical Society, vol. 333, no. 1, pp. 177-190, 2002.

[84] S. E. Koposov, J. Yoo, H.-W. Rix, D. H. Weinberg, A. V. Macciò, and J. M. Escudé, "A quantitative explanation of the observed population of Milky Way satellite galaxies," The Astrophysical Journal, vol. 696, no. 2, pp. 2179-2194, 2009.

[85] Y.-S. Li, A. Helmi, G. De Lucia, and F. Stoehr, "On the common mass scale of the Milky Way satellites," Monthly Notices of the Royal Astronomical Society, vol. 397, no. 1, pp. L87-L91, 2009.

[86] A. V. Macciò, X. Kang, and B. Moore, "Central mass and luminosity of Milky Way satellites in the $\lambda$ cold dark matter model," The Astrophysical Journal, vol. 692, no. 2, pp. L109L112, 2009.

[87] M. T. Busha, M. A. Alvarez, R. H. Wechsler, T. Abel, and L. E. Strigari, "The impact of inhomogeneous reionization on the satellite galaxy population of the Milky Way," http://arxiv.org/abs/0901.3553.

[88] L. E. Strigari, J. S. Bullock, M. Kaplinghat, et al., "A common mass scale for satellite galaxies of the Milky Way," Nature, vol. 454, no. 7208, pp. 1096-1097, 2008.

[89] F. Stoehr, S. D. M. White, G. Tormen, and V. Springel, "The satellite population of the Milky Way in a ACDM universe," Monthly Notices of the Royal Astronomical Society, vol. 335, no. 4, pp. L84-L88, 2002.

[90] L. E. Strigari, J. S. Bullock, M. Kaplinghat, J. Diemand, M. Kuhlen, and P. Madau, "Redefining the missing satellites problem,” The Astrophysical Journal, vol. 669, no. 2, pp. 676683, 2007.

[91] E. Hayashi, J. F. Navarro, J. E. Taylor, J. Stadel, and T. Quinn, "The structural evolution of substructure," The Astrophysical Journal, vol. 584, no. 2, pp. 541-558, 2003.

[92] S. Kazantzidis, L. Mayer, C. Mastropietro, J. Diemand, J. Stadel, and B. Moore, "Density profiles of cold dark matter substructure: implications for the missing-satellites problem," The Astrophysical Journal, vol. 608, no. 2, pp. 663679, 2004.

[93] O. Y. Gnedin, A. V. Kravtsov, A. A. Klypin, and D. Nagai, "Response of dark matter halos to condensation of baryons: cosmological simulations and improved adiabatic contraction model," The Astrophysical Journal, vol. 616, no. 1, pp. 16-26, 2004.

[94] J. Diemand, M. Kuhlen, and P. Madau, "Formation and evolution of galaxy dark matter halos and their substructure," The Astrophysical Journal, vol. 667, no. 2, pp. 859-877, 2007.

[95] A. R. Zentner, A. A. Berlind, J. S. Bullock, A. V. Kravtsov, and R. H. Wechsler, "The physics of galaxy clustering. I. A model for subhalo populations," The Astrophysical Journal, vol. 624, no. 2, pp. 505-525, 2005.

[96] M. Colpi, L. Mayer, and F. Governato, "Dynamical friction and the evolution of satellites in virialized halos: the theory of linear response," The Astrophysical Journal, vol. 525, no. 2, pp. 720-733, 1999.

[97] Y. Hashimoto, Y. Funato, and J. Making, "To circularize or not to circularize?-Orbital evolution of satellite galaxies," The Astrophysical Journal, vol. 582, no. 1, pp. 196-201, 2003.

[98] M. Boylan-Kolchin, C.-P. Ma, and E. Quataert, "Dynamical friction and galaxy merging time-scales," Monthly Notices of the Royal Astronomical Society, vol. 383, no. 1, pp. 93-101, 2008.

[99] C. Y. Jiang, Y. P. Jing, A. Faltenbacher, W. P. Lin, and C. Li, "A fitting formula for the merger timescale of galaxies in hierarchical clustering," The Astrophysical Journal, vol. 675, no. 2, pp. 1095-1105, 2008.

[100] S. Naoz, R. Barkana, and A. Mesinger, "Gas in simulations of high redshift galaxies and minihalos," Monthly Notices of the Royal Astronomical Society, vol. 399, p. 369, 2009, http://adsabs.harvard.edu/abs/2009MNRAS.399..369N.

[101] T. Abel, G. L. Bryan, and M. L. Norman, "The formation of the first star in the universe," Science, vol. 295, no. 5552, pp. 93-98, 2002.

[102] J. E. Taylor, J. Silk, and A. Babul, "Clues to dwarf galaxy formation from clustering and kinematics," in Near-Fields Cosmology with Dwarf Elliptical Galaxies, H. Jerjen and B. Binggeli, Eds., vol. 198 of IAU Symposium and Colloquium, pp. 185-188, CUP, Cambridge, UK, 2005.

[103] E. D'Onghia and G. Lake, "Small dwarf galaxies within larger dwarfs: why some are luminous while most go dark," The Astrophysical Journal, vol. 686, no. 2, pp. L61-L65, 2008.

[104] J. Bailin, C. Power, P. Norberg, D. Zaritsky, and B. K. Gibson, "The anisotropic distribution of satellite galaxies," Monthly Notices of the Royal Astronomical Society, vol. 390, no. 3, pp. 1133-1156, 2008.

[105] J. Chen, A. V. Kravtsov, F. Prada, et al., "Constraining the projected radial distribution of galactic satellites with the sloan digital sky survey," The Astrophysical Journal, vol. 647, no. 1, pp. 86-101, 2006.

[106] M. Kamionkowski and A. R. Liddle, "The dearth of halo dwarf galaxies: is there power on short scales?" Physical Review Letters, vol. 84, no. 20, pp. 4525-4528, 2000. 
[107] P. Colín, V. Avila-Reese, and O. Valenzuela, "Substructure and halo density profiles in a warm dark matter cosmology," The Astrophysical Journal, vol. 542, no. 2, pp. 622-630, 2000.

[108] P. Bode, J. P. Ostriker, and N. Turok, "Halo formation in warm dark matter models," The Astrophysical Journal, vol. 556, no. 1, pp. 93-107, 2001.

[109] P. Colín, O. Valenzuela, and V. Avila-Reese, "On the structure of dark matter halos at the damping scale of the power spectrum with and without relict velocities," The Astrophysical Journal, vol. 673, no. 1, pp. 203-214, 2008.

[110] D. N. Spergel and P. J. Steinhardt, "Observational evidence for self-interacting cold dark matter," Physical Review Letters, vol. 84, no. 17, pp. 3760-3763, 2000.

[111] B. Moore, S. Gelato, A. Jenkins, F. R. Pearce, and V. Quilis, "Collisional versus collisionless dark matter," The Astrophysical Journal, vol. 535, no. 1, pp. L21-L24, 2000.

[112] C. S. Kochanek and M. White, "A quantitative study of interacting dark matter in halos," The Astrophysical Journal, vol. 543, no. 2, pp. 514-520, 2000.

[113] N. Yoshida, V. Springel, S. D. M. White, and G. Tormen, "Weakly self-interacting dark matter and the structure of dark halos," The Astrophysical Journal, vol. 544, no. 2, pp. L87-L90, 2000.

[114] J. Miralda-Escudé, "A test of the collisional dark matter hypothesis from cluster lensing," The Astrophysical Journal, vol. 564, no. 1, pp. 60-64, 2002.

[115] O. Y. Gnedin and J. P. Ostriker, "Limits on collisional dark matter from elliptical galaxies in clusters," The Astrophysical Journal, vol. 561, no. 1, pp. 61-68, 2001.

[116] D. Clowe, M. Bradač, A. H. Gonzalez, et al., "A direct empirical proof of the existence of dark matter," The Astrophysical Journal, vol. 648, no. 2, pp. L109-L113, 2006.

[117] K. Abazajian, "Linear cosmological structure limits on warm dark matter," Physical Review D, vol. 73, no. 6, Article ID 063513, 6 pages, 2006.

[118] U. Seljak, A. Makarov, P. McDonald, and H. Trac, "Can sterile neutrinos be the dark matter?" Physical Review Letters, vol. 97, no. 19, Article ID 191303, 4 pages, 2006.

[119] S. Mao and P. Schneider, "Evidence for substructure in lens galaxies?" Monthly Notices of the Royal Astronomical Society, vol. 295, no. 3, pp. 587-594, 1998.

[120] N. Dalal and C. S. Kochanek, "Direct detection of cold dark matter substructure," The Astrophysical Journal, vol. 572, no. 1, pp. 25-33, 2002.

[121] C. S. Kochanek and N. Dalal, "Tests for substructure in gravitational lenses," The Astrophysical Journal, vol. 610, no. 1, pp. 69-79, 2004.

[122] S. D. M. White and C. S. Frenk, "Galaxy formation through hierarchical clustering," The Astrophysical Journal, vol. 379, no. 1 , pp. 52-79, 1991.

[123] A. H. Gonzalez, K. A. Williams, J. S. Bullock, T. S. Kolatt, and J. R. Primack, "The velocity function of galaxies," The Astrophysical Journal, vol. 528, no. 1, pp. 145-155, 2000.

[124] A. J. Benson, R. G. Bower, C. S. Frenk, C. G. Lacey, C. M. Baugh, and S. Cole, "What shapes the luminosity function of galaxies?" The Astrophysical Journal, vol. 599, no. 1, pp. 3849, 2003.

[125] I. K. Baldry, K. Glazebrook, and S. P. Driver, "On the galaxy stellar mass function, the mass-metallicity relation and the implied baryonic mass function," Monthly Notices of the Royal Astronomical Society, vol. 388, no. 3, pp. 945-959, 2008.

[126] A. V. Tikhonov and A. Klypin, "The emptiness of voids: yet another overabundance problem for the $\Lambda$ cold dark matter model," Monthly Notices of the Royal Astronomical Society, vol. 395, no. 4, pp. 1915-1924, 2009.

[127] A. A. Thoul and D. H. Weinberg, "Hydrodynamic simulations of galaxy formation. II. Photoionization and the formation of low-mass galaxies," The Astrophysical Journal, vol. 465, no. 2, pp. 608-616, 1996.

[128] T. Quinn, N. Katz, and G. Efstathiou, "Photoionization and the formation of dwarf galaxies," Monthly Notices of the Royal Astronomical Society, vol. 278, no. 4, pp. L49-L54, 1996.

[129] N. Y. Gnedin and L. Hui, "Probing the Universe with the Ly $\alpha$ forest-I. Hydrodynamics of the low-density intergalactic medium," Monthly Notices of the Royal Astronomical Society, vol. 296, no. 1, pp. 44-55, 1998.

[130] T. Kitayama and S. Ikeuchi, "Formation of subgalactic clouds under ultraviolet background radiation," The Astrophysical Journal, vol. 529, no. 2, pp. 615-634, 2000.

[131] N. Y. Gnedin, "Effect of reionization on structure formation in the universe," The Astrophysical Journal, vol. 542, no. 2, pp. 535-541, 2000.

[132] M. Dijkstra, Z. Haiman, M. J. Rees, and D. H. Weinberg, "Photoionization feedback in low-mass galaxies at high redshift," The Astrophysical Journal, vol. 601, no. 2, pp. 666675, 2004.

[133] M. Hoeft, G. Yepes, S. Gottlöber, and V. Springel, "Dwarf galaxies in voids: suppressing star formation with photoheating," Monthly Notices of the Royal Astronomical Society, vol. 371, no. 1, pp. 401-414, 2006.

[134] T. Okamoto, L. Gao, and T. Theuns, "Mass loss of galaxies due to an ultraviolet background," Monthly Notices of the Royal Astronomical Society, vol. 390, no. 3, pp. 920-928, 2008.

[135] R. Barkana and A. Loeb, "The photoevaporation of dwarf galaxies during reionization," The Astrophysical Journal, vol. 523, no. 1, pp. 54-65, 1999.

[136] N. J. Shaviv and A. Dekel, "Photo-evaporation by thermal winds in dwarf galaxies," submitted to Monthly Notices of the Royal Astronomical Society.

[137] P. R. Shapiro, I. T. Iliev, and A. C. Raga, "Photoevaporation of cosmological minihaloes during reionization," Monthly Notices of the Royal Astronomical Society, vol. 348, no. 3, pp. 753-782, 2004.

[138] A. Dekel and J. Silk, "The origin of dwarf galaxies, cold dark matter, and biased galaxy formation," The Astrophysical Journal, vol. 303, pp. 39-55, 1986.

[139] M.-M. Mac Low and A. Ferrara, "Starburst-driven mass loss from dwarf galaxies: efficiency and metal ejection," The Astrophysical Journal, vol. 513, no. 1, pp. 142-155, 1999.

[140] A. Dekel and J. Woo, "Feedback and the fundamental line of low-luminosity low-surface-brightness/dwarf galaxies," Monthly Notices of the Royal Astronomical Society, vol. 344, no. 4, pp. 1131-1144, 2003.

[141] S. Mashchenko, J. Wadsley, and H. M. P. Couchman, "Stellar feedback in dwarf galaxy formation," Science, vol. 319, no. 5860, pp. 174-177, 2008.

[142] A. Marcolini, A. D’Ercole, F. Brighenti, and S. Recchi, “Star formation feedback and metal enrichment by types Ia and II supernovae in dwarf spheroidal galaxies: the case of Draco," Monthly Notices of the Royal Astronomical Society, vol. 371, no. 2, pp. 643-658, 2006.

[143] Z. Haiman, M. J. Rees, and A. Loeb, "Destruction of molecular hydrogen during cosmological reionization," The Astrophysical Journal, vol. 476, no. 2, pp. 458-463, 1997.

[144] Z. Haiman, T. Abel, and M. J. Rees, "The radiative feedback of the first cosmological objects," The Astrophysical Journal, vol. 534, no. 1, pp. 11-24, 2000. 
[145] E. Scannapieco, R. J. Thacker, and M. Davis, "High-redshift galaxy outflows and the formation of dwarf galaxies," The Astrophysical Journal, vol. 557, no. 2, pp. 605-615, 2001.

[146] F. Sigward, A. Ferrara, and E. Scannapieco, "Suppression of dwarf galaxy formation by cosmic shocks," Monthly Notices of the Royal Astronomical Society, vol. 358, no. 3, pp. 755-764, 2005.

[147] T. Kaufmann, C. Wheeler, and J. S. Bullock, "On the morphologies, gas fractions, and star formation rates of small galaxies," Monthly Notices of the Royal Astronomical Society, vol. 382, no. 3, pp. 1187-1195, 2007.

[148] K. Tassis, A. V. Kravtsov, and N. Y. Gnedin, "Scaling relations of dwarf galaxies without supernova-driven winds," The Astrophysical Journal, vol. 672, no. 2, pp. 888-903, 2008.

[149] B. E. Robertson and A. V. Kravtsov, "Molecular hydrogen and global star formation relations in galaxies," The Astrophysical Journal, vol. 680, no. 2, pp. 1083-1111, 2008.

[150] N. Y. Gnedin, K. Tassis, and A. V. Kravtsov, "Modeling molecular hydrogen and star formation in cosmological simulations," The Astrophysical Journal, vol. 697, no. 1, pp. $55-67,2009$.

[151] N. Y. Gnedin and A. V. Kravtsov, "Fossils of reionization in the local group," The Astrophysical Journal, vol. 645, no. 2, pp. 1054-1061, 2006.

[152] B. Moore, J. Diemand, P. Madau, M. Zemp, and J. Stadel, "Globular clusters, satellite galaxies and stellar haloes from early dark matter peaks," Monthly Notices of the Royal Astronomical Society, vol. 368, no. 2, pp. 553-570, 2006.

[153] P. Madau, M. Kuhlen, J. Diemand, et al., "Fossil remnants of reionization in the halo of the Milky Way," The Astrophysical Journal, vol. 689, no. 1, pp. L41-L44, 2008.

[154] M. Ricotti and N. Y. Gnedin, "Formation histories of dwarf galaxies in the Local Group," The Astrophysical Journal, vol. 629, no. 1, pp. 259-267, 2005.

[155] M. S. Bovill and M. Ricotti, "Pre-reionization fossils, ultrafaint dwarfs and the missing galactic satellite problem," The Astrophysical Journal, vol. 693, no. 2, pp. 1859-1870, 2009.

[156] M. Ricotti, Advances in Astronomy, vol. 2009, 2009.

[157] F. Stoehr, S. D. M. White, V. Springel, G. Tormen, and N. Yoshida, "Dark matter annihilation in the halo of the Milky Way," Monthly Notices of the Royal Astronomical Society, vol. 345, no. 4, pp. 1313-1322, 2003.

[158] S. Salvadori, A. Ferrara, and R. Schneider, "Life and times of dwarf spheroidal galaxies," Monthly Notices of the Royal Astronomical Society, vol. 386, no. 1, pp. 348-358, 2008.

[159] E. K. Grebel, J. S. Gallagher III, and D. Harbeck, "The progenitors of dwarf spheroidal galaxies," The Astronomical Journal, vol. 125, no. 4, pp. 1926-1939, 2003.

[160] Y. Fenner, B. K. Gibson, R. Gallino, and M. Lugaro, "Cosmological implications of dwarf spheroidal chemical evolution," The Astrophysical Journal, vol. 646, no. 1, pp. 184191, 2006.

[161] J. I. Read, A. P. Pontzen, and M. Viel, "On the formation of dwarf galaxies and stellar haloes," Monthly Notices of the Royal Astronomical Society, vol. 371, no. 2, pp. 885-897, 2006.

[162] S. Salvadori and A. Ferrara, "Ultra faint dwarfs: probing early cosmic star formation," Monthly Notices of the Royal Astronomical Society, vol. 395, no. 1, pp. L6-L10, 2009.

[163] M. Mateo, E. W. Olszewski, C. Pryor, D. L. Welch, and P. Fischer, "The Carina dwarf spheroidal galaxy: how dark is it?" The Astronomical Journal, vol. 105, no. 2, pp. 510-526, 1993.

[164] G. Gilmore, M. I. Wilkinson, R. F. G. Wyse, et al., "The observed properties of dark matter on small spatial scales," The Astrophysical Journal, vol. 663, no. 2, pp. 948-959, 2007.
[165] J. S. Bullock, T. S. Kolatt, Y. Sigad, et al., "Profiles of dark haloes: evolution, scatter and environment," Monthly Notices of the Royal Astronomical Society, vol. 321, no. 3, pp. 559-575, 2001.

[166] C. Conroy, R. H. Wechsler, and A. V. Kravtsov, "Modeling luminosity-dependent galaxy clustering through cosmic time," The Astrophysical Journal, vol. 647, no. 1, pp. 201-214, 2006.

[167] C. Conroy and R. H. Wechsler, "Connecting galaxies, halos, and star formation rates across cosmic time," The Astrophysical Journal, vol. 696, no. 1, pp. 620-635, 2009.

[168] J. L. Tinker and C. Conroy, "The void phenomenon explained," The Astrophysical Journal, vol. 691, pp. 633-639, 2009.

[169] R. Braun and W. B. Burton, "The kinematic and spatial deployment of compact, isolated high-velocity clouds," Astronomy \& Astrophysics, vol. 341, no. 2, pp. 437-450, 1999.

[170] V. de Heij, R. Braun, and W. B. Burton, "An all-sky study of compact, isolated high-velocity clouds," Astronomy \& Astrophysics, vol. 392, no. 2, pp. 417-451, 2002.

[171] D. A. Thilker, R. Braun, R. A. M. Walterbos, et al., "On the continuing formation of the Andromeda galaxy: detection of H I clouds in the M31 halo," The Astrophysical Journal, vol. 601, no. 1, pp. L39-L42, 2004.

[172] M. Grossi, C. Giovanardi, E. Corbelli, et al., "H I clouds in the proximity of M 33," Astronomy \& Astrophysics, vol. 487, no. 1, pp. 161-175, 2008.

[173] F. C. van den Bosch, X. Yang, H. J. Mo, et al., "Towards a concordant model of halo occupation statistics," Monthly Notices of the Royal Astronomical Society, vol. 376, no. 2, pp. 841-860, 2007.

[174] N. F. Martin, J. T. A. de Jong, and H.-W. Rix, "A comprehensive maximum likelihood analysis of the structural properties of faint Milky Way satellites," The Astrophysical Journal, vol. 684, no. 2, pp. 1075-1092, 2008.

[175] J. Pizagno, F. Prada, D. H. Weinberg, et al., "Dark matter and stellar mass in the luminous regions of disk galaxies," The Astrophysical Journal, vol. 633, no. 2, pp. 844-856, 2005.

[176] R. Mandelbaum, U. Seljak, G. Kauffmann, C. M. Hirata, and J. Brinkmann, "Galaxy halo masses and satellite fractions from galaxy-galaxy lensing in the Sloan Digital Sky Survey: stellar mass, luminosity, morphology and environment dependencies," Monthly Notices of the Royal Astronomical Society, vol. 368, no. 2, pp. 715-731, 2006.

[177] C. Conroy, F. Prada, J. A. Newman, et al., "Evolution in the halo masses of isolated galaxies between $z \sim 1$ and $z \sim 0$ : from DEEP2 to SDSS," The Astrophysical Journal, vol. 654, no. 1, pp. 153-171, 2007.

[178] A. Klypin, F. Prada, and A. Montero-Dorta, submitted to Astrophysical Journal. 

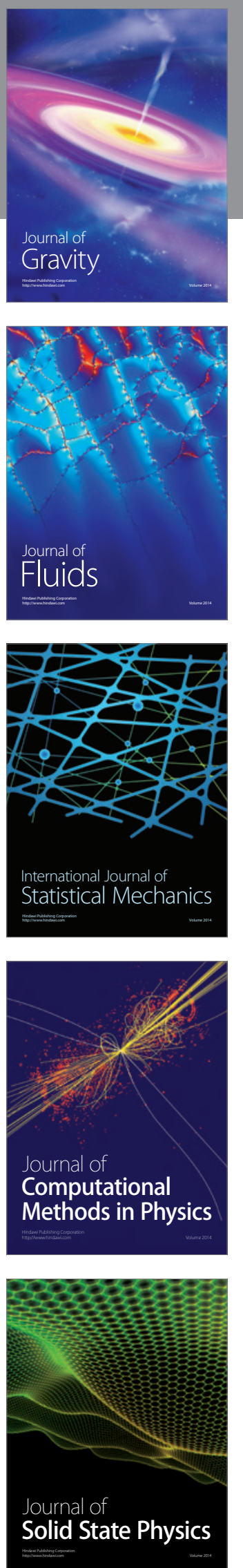

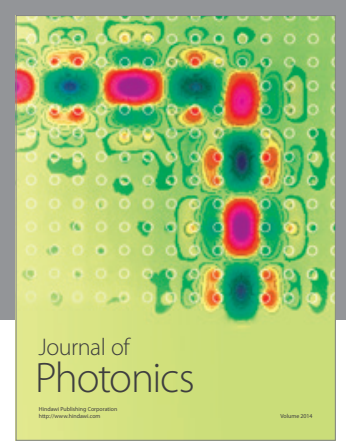

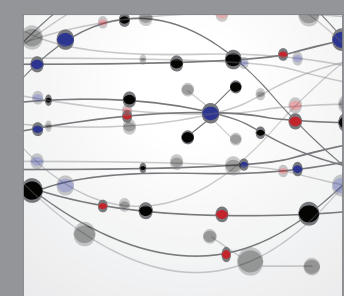

The Scientific World Journal
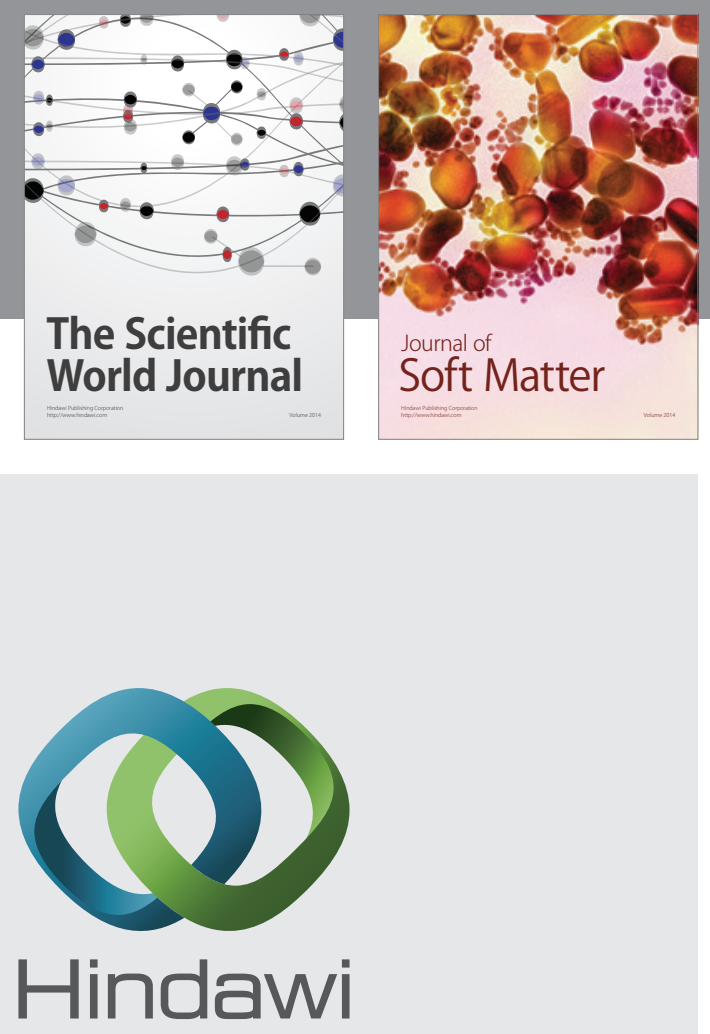

Submit your manuscripts at

http://www.hindawi.com
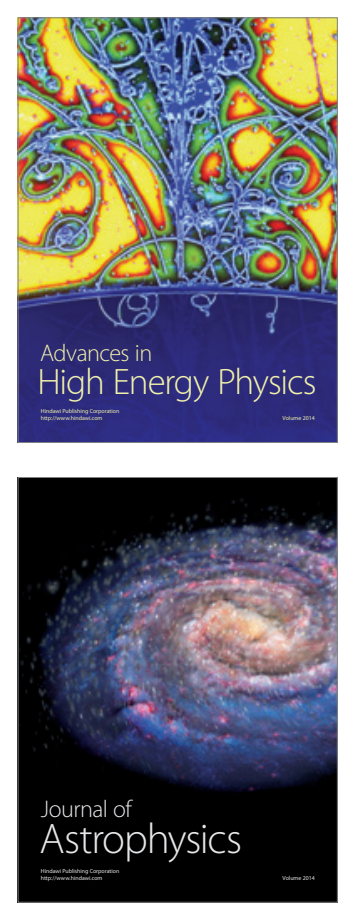
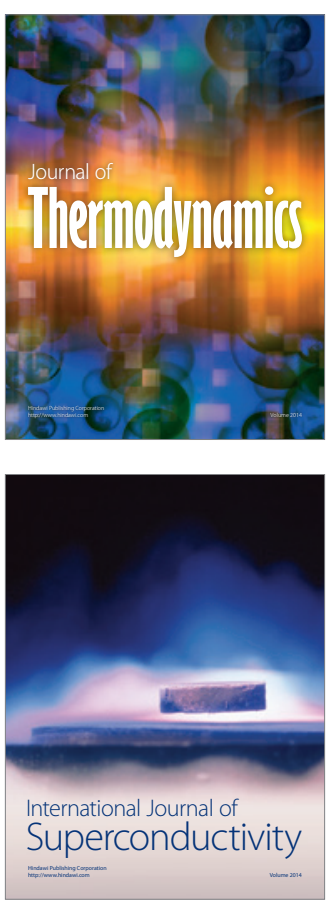
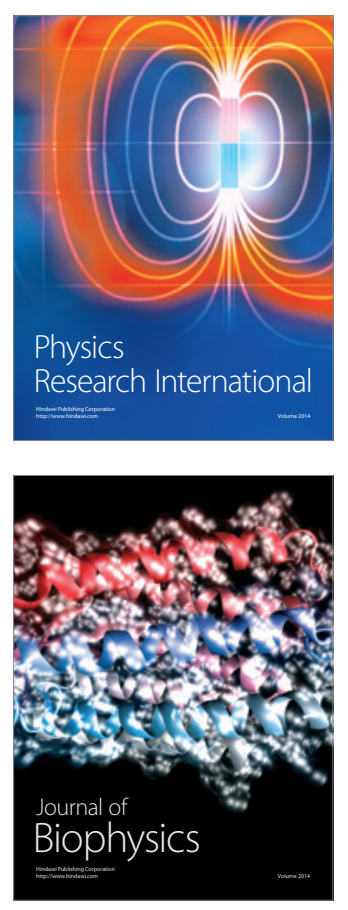
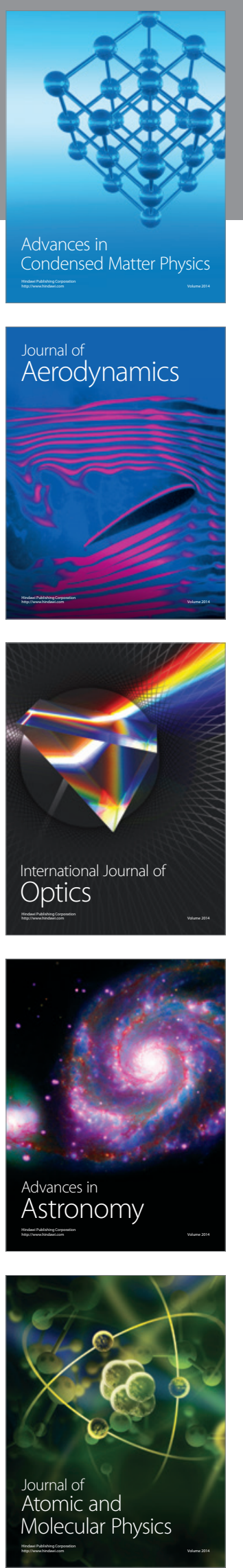